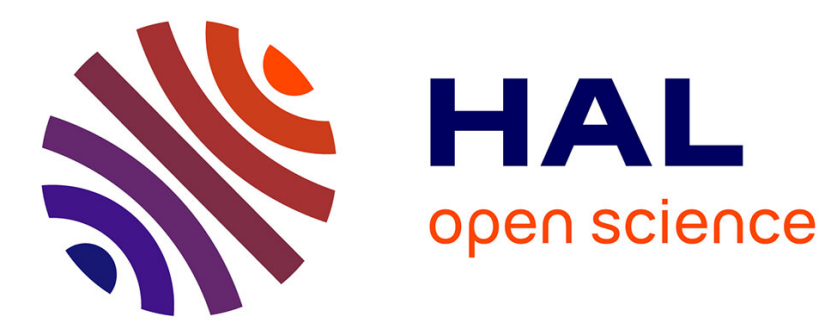

\title{
Progress towards ultrafast spintronics applications
}

\author{
Amal El-Ghazaly, J. Gorchon, Richard B. Wilson, Akshay Pattabi, Jeffrey
}

Bokor

\section{To cite this version:}

Amal El-Ghazaly, J. Gorchon, Richard B. Wilson, Akshay Pattabi, Jeffrey Bokor. Progress towards ultrafast spintronics applications. Journal of Magnetism and Magnetic Materials, 2020, 502, pp.166478. 10.1016/j.jmmm.2020.166478 . hal-02901699

\section{HAL Id: hal-02901699 \\ https://hal.univ-lorraine.fr/hal-02901699}

Submitted on 17 Jul 2020

HAL is a multi-disciplinary open access archive for the deposit and dissemination of scientific research documents, whether they are published or not. The documents may come from teaching and research institutions in France or abroad, or from public or private research centers.
L'archive ouverte pluridisciplinaire $\mathbf{H A L}$, est destinée au dépôt et à la diffusion de documents scientifiques de niveau recherche, publiés ou non, émanant des établissements d'enseignement et de recherche français ou étrangers, des laboratoires publics ou privés. 


\section{Journal Pre-proofs}

Progress Towards Ultrafast Spintronics Applications

Amal El-Ghazaly, Jon Gorchon, Richard B. Wilson, Akshay Pattabi, Jeffrey

Bokor

PII:

S0304-8853(19)32672-1

DOI:

https://doi.org/10.1016/j.jmmm.2020.166478

Reference:

MAGMA 166478

To appear in:

Journal of Magnetism and Magnetic Materials

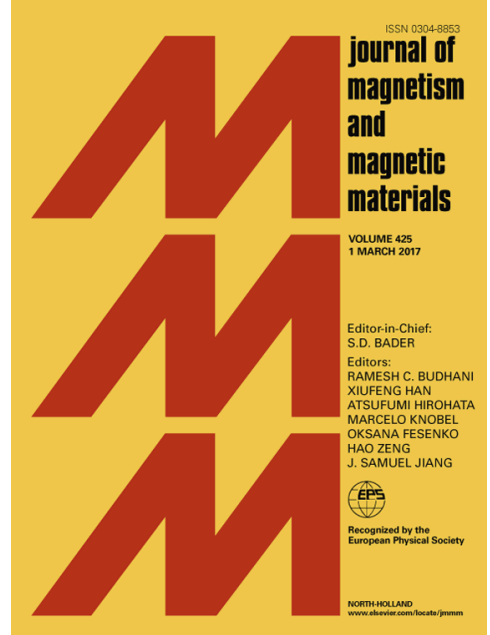

Revised Date: $\quad 24$ December 2019

Accepted Date: $\quad 17$ January 2020

Please cite this article as: A. El-Ghazaly, J. Gorchon, R.B. Wilson, A. Pattabi, J. Bokor, Progress Towards Ultrafast Spintronics Applications, Journal of Magnetism and Magnetic Materials (2020), doi: https://doi.org/10.1016/ j.jmmm.2020.166478

This is a PDF file of an article that has undergone enhancements after acceptance, such as the addition of a cover page and metadata, and formatting for readability, but it is not yet the definitive version of record. This version will undergo additional copyediting, typesetting and review before it is published in its final form, but we are providing this version to give early visibility of the article. Please note that, during the production process, errors may be discovered which could affect the content, and all legal disclaimers that apply to the journal pertain. 


\title{
Progress Towards Ultrafast Spintronics Applications
}

\author{
Amal El-Ghazaly \\ School of Electrical and Computer Engineering, Cornell University, Ithaca, NY 14853, \\ $U S A$ \\ Jon Gorchon \\ Université de Lorraine, CNRS, IJL, F-54000 Nancy, France \\ Richard B. Wilson \\ Department of Mechanical Engineering, University of California, Riverside, CA 92521, \\ USA \\ Akshay Pattabi, Jeffrey Bokor \\ Department of Electrical Engineering and Computer Science, University of California, \\ Berkeley, CA 94720, USA
}

\begin{abstract}
The discovery of ultrafast demagnetization by Bigot and Beaurepaire in 1996 [1] launched the field of ultrafast magnetism - the study of subpicosecond magnetization dynamics in response to femtosecond laser excitations. In the same year, the discovery of spin-transfer torque switching of magnetic nanostructures [2,3] launched the field of spintronics - the control of magnetic order with electrical currents and voltage, germane to integrated electronic systems. Ultrafast magnetism may be particularly useful to future spintronic memory and logic devices by enabling magnetization switching at much faster time scales than in any existing spintronic technology. However, a number of obstacles stand in the way of integrating ultrafast magnetic phenomena into spintronic devices. To be useful for devices, ultrafast magnetization dynamics must be driven by electrical currents, not femtosecond lasers. The electrical currents need to be sourced by semiconductor transistors, which currently have a minimum gate delay on the order of picoseconds. Readout of the magnetic state must also be electrical, which will likely require a large magnetoresistance from a ferromagnetic tunnel junction. Finally, the
\end{abstract}


switching energy must be minimized, which requires nanoscale device dimensions.

This review discusses our most recent advances in addressing these challenges and bridging together the two fields of spintronics and ultrafast magnetism to enable the integration of ultrafast spintronic devices. Our research shows that, not only sub-picosecond optical pulses, but also picosecond excitations in the form of optical and electrical (heat current) pulses can result in the switching of the magnetization direction between two opposite states in ferrimagnetic GdFeCo. We also demonstrate ultrafast single shot switching of ferromagnetic $\mathrm{Co} / \mathrm{Pt}$ exchange coupled to a GdFeCo layer. Furthermore, we observe ultrafast switching in nanoscale patterned GdCo dots. These achievements pave the way towards the construction of nanoscale ferromagnetic tunnel junctions capable of picosecond magnetization write times and electrical readout.

Keywords: ultrafast, all-optical switching, sub-picosecond, GdFeCo, GdCo, ultrafast spintronics

\section{Introduction}

In 1996, Jean-Yves Bigot and Eric Beaurepaire revealed sub-picosecond demagnetization behavior of ferromagnetic nickel in response to a femtosecond laser pulse excitation [1]. Their pioneering discovery has since inspired many new research directions focused on ultrafast magnetization dynamics. Perhaps the result most relevant to electronics applications was the achievement of picosecond magnetization reversal of the GdFeCo alloy in $2007[4,5]$. Although, these initial results seemed to suggest that the reversal of magnetization depended on the helicity of the circularly-polarized laser pulse excitation $[4,5,6]$, subsequent studies revealed that linearly-polarized light also drives ultrafast toggle switching $[7,8,9,10]$, presented in Fig. 1. An ability to drive switching with a linearly polarized laser pulse suggests that the phenomena is primarily heat driven. Magneto-optical phenomena are of secondary importance [11]. Equipped with this understanding that helicityindependent all-optical switching (HI-AOS) in ferrimagnetic metals is a heatdriven phenomenon, numerous research efforts have begun to explore different methods of supplying that heat without the use of a femtosecond laser $[12,13,14,15,16,17]$. Together with other research efforts $[18,19,20]$, significant progress is being made toward integrating ultrafast switching into 

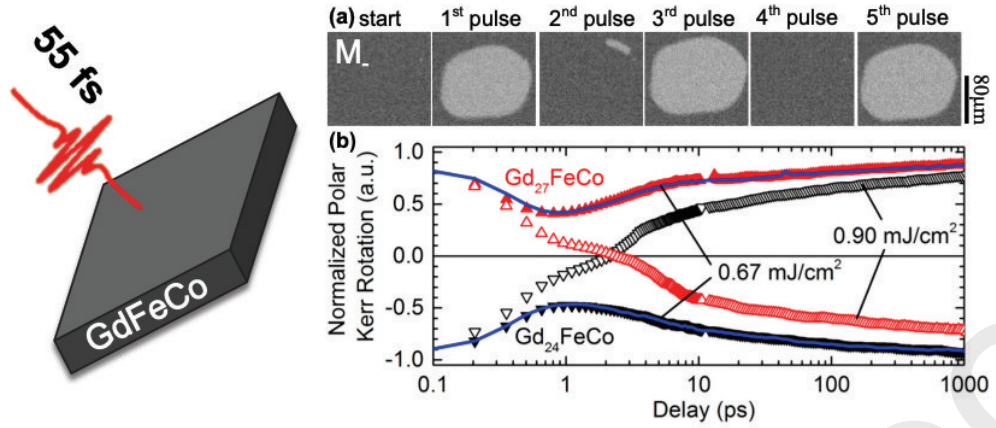

Figure 1: (a) MOKE micrographs of the magnetization of the $\mathrm{Gd}_{27} \mathrm{FeCo}$ film exposed to successive linearly polarized pulses of $t=55$ fs on an initially "down" (M-) magnetized sample. Reliable all-optical switching of the magentization independly of the helicity of light is demonstrated. (b) Evolution of the normalized Kerr rotation of GdFeCo samples induced by a linearly polarized $t=55$ fs pump. Adapted figure with permission from [21]. Copyright 2016 by the American Physical Society.

electronic devices $[12,13,14,15,16,17,18,19,20]$.

Integration of spintronic devices with conventional electronics requires that both the control and detection be done using basic integrated circuit parameters, like current, voltage, and resistance. Giant magnetoresistance (GMR) $[22,23]$ and tunneling magnetoresistance (TMR) $[24,25,26,27,28$, $29,30]$ allow for the detection of spin in terms of a simple resistance measurement between ferromagnetic layers [31]. As a result, magnetoresistance has become the standard method for "reading" the magnetic state. On the other hand, multiple different types of methods are currently being explored for "writing" the magnetic state.

To fulfill similar data storage functions as integrated semiconductor technologies like SRAM and DRAM [32], rising magnetic random access memory (MRAM) technologies must satisfy certain performance specifications. These include short read and write times, low dynamic energy, low static/leakage current, and small device area/high density [33, 34]. For MRAM devices, these specifications correspond to the following criteria and performance metrics:

- Fast switching (for short write times)

- Low switching energy (for low dynamic energy)

- Nonvolatility (inherent to magnetic materials and minimizes static leak- 
age)

- Nanoscale integration (while maintaining sufficiently large magnetic anisotropy to provide thermal stability for small devices and higher densities)

In addition, we can include the previously mentioned criteria that the magnetic device should be written and read by the basic electronic circuit parameters - current, voltage, and resistance.

All MRAM technologies are inherently nonvolatile and can generally be scaled down to nanometer dimensions. Therefore, the remaining two performance metrics for comparing magnetization switching technologies are switching energy per unit area (of the magnetic material) and switching speed. Devices that can minimize both energy consumption and speed are the most attractive for applications. Using these metrics, Fig. 2 compares ultrafast magnetization switching in uniaxial ferrimagnetic metals, which is the focus of this review, with the most prominent existing magnetization switching technologies, those of spin-transfer torque (STT), spin-orbit torque (SOT), and magnetoelectric/voltage-controlled (ME) switching. As can be seen from the figure, successful integration of ultrafast magnetization dynamics for spintronics applications has the potential to provide the fastest switching speed while still consuming a reasonably low switching energy, as estimated by [14].

Thanks to Bigot and Beaurepaire, the discovery of sub-picosecond magnetization dynamics has opened the door to numerous new research pursuits in all-optical switching, terahertz spintronics and ultrafast magnetic memory and logic devices [48, 49]. The main challenges facing the field currently include gaining a better understanding of the physics behind the switching process and making progress towards complete integration of ultrafast spintronic devices [20]. This review highlights some of our research efforts attempting to address those challenges. In Section 2, we discuss our experimental observations of magnetization dynamics in response to excitations of different pulse widths and energies. Femtosecond pulse experiments show that the energy required for ultrafast switching of $\mathrm{GdFeCo}$ is quite low. Furthermore, the energy required for switching depends only weakly on the pulse duration. This is in stark contrast with STT and SOT, whose required switching energy dramatically increases for pulses shorter than one nanosecond [35, 36, 39, 41, 42, 43]. In the same section, we also discuss 


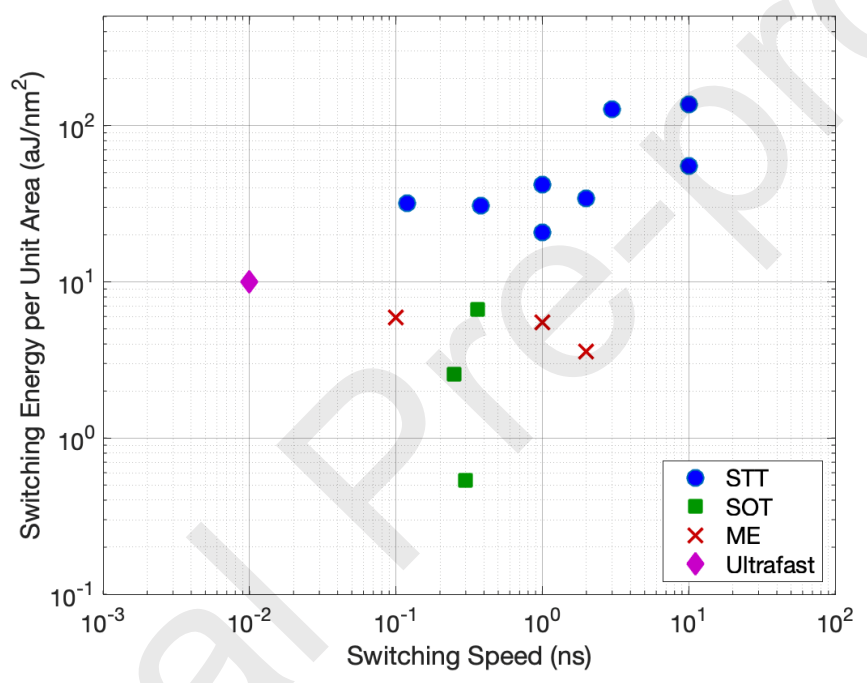

Figure 2: Summary of existing magnetization switching technologies for MRAM as well as the potential technology of ultrafast magnetization switching. The comparison is made based upon reported switching energy per unit area and switching speed from [35, 36, $37,38,39,40,41,42,43,44,45,46,47]$ compared to the estimated values for ultrafast electrical switching from [14]. 
how hot electron diffusion from an adjacent metal is sufficient to cause ultrafast magnetization switching in GdFeCo. Then in Section 3, we present the ability of picosecond electrical current pulses to drive ultrafast magnetization dynamics. Time-resolved measurements of magnetization dynamics confirm that electrical heating can both demagnetize ferromagnetic $\mathrm{Co} / \mathrm{Pt}$ multilayers and switch ferrimagnetic GdFeCo. Afterwards, in Section 4, we attempt to confront various roadblocks to ultrafast spintronic integration by, first, demonstrating ultrafast magnetic reversal of a ferromagnet, which can be used in constructing magnetoresistive tunnel junctions for electrical readout. Second, we fabricate and measure nanoscale ferrimagnetic patterns and demonstrate that HI-AOS phenomena are not only maintained down to nanoscale dimensions, but are, in fact, enhanced at such dimensions. Finally, we conclude this review with some insights about the impact of ultrafast magnetization reversal as well as potential directions for future research.

\section{Picosecond heat pulses for magnetization switching}

Although ultrafast all-optical switching (AOS) has been observed in a number of insulating films $[50,51,52]$ in addition to uniaxial ferrimagnetic metals like the GdFeCo we describe in this review, it is widely accepted that in these latter systems, AOS is observed as a $180^{\circ}$ reversal of its two antiparallel magnetic sub-systems $[53,9,10,54]$. So far, ultrafast AOS has only been observed in ferrimagnetic Gd and Co or Fe based alloys [9, 55, 56], and more recently in multilayers [57, 17], independent of the helicity of the optical excitation. Moreover, it is also believed that, in a material film, these magnetic subsystems must be allowed to exchange angular momentum with each other upon laser excitation in order to reverse the direction of their magnetic moment. In fact, the key to HI-AOS resides in the fact that the angular momentum loss from one of the subsystems (e.g., Gd) during its demagnetization will be partially absorbed by the other subsystems (e.g., FeCo), eventually driving it to switch.

In the case of GdFeCo alloys, it was experimentally observed that the FeCo sublattice demagnetizes faster than the Gd one, crosses zero, and becomes transiently parallel to the Gd sublattice [9]. To explain such surprising behavior, it was put forth by $[9,58]$ that the electronic temperature had to reach a much higher temperature than $T_{c}$ so as to temporarily "break" the exchange interaction and allow the sublattices to decouple and demagnetize at different rates. If such a condition was found to be true, it would mean 
(a)

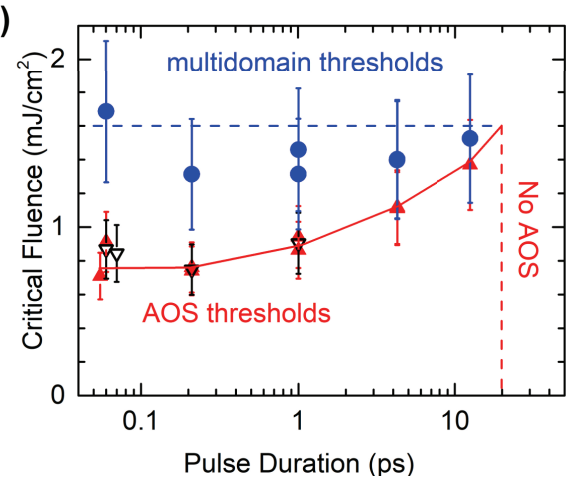

(b)

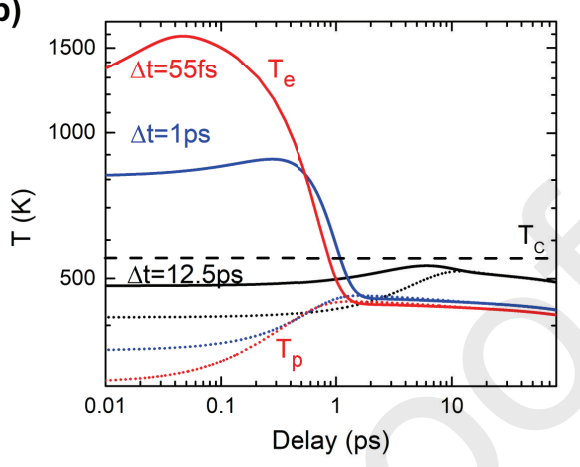

Figure 3: (a) Critical fluence $\mathrm{F}_{\mathrm{c}}$ for $\mathrm{AOS}$ and multidomain states as a function of the laser pulse duration at room temperature. Solid lines are guides for the eyes. The blue dashed lines are a calculation of the fluence needed to make the lattice reach $T_{c}$. The magneto-optic Kerr effect (MOKE) image shows the limits for observation of AOS in each sample. The fully demagnetized state obtained for a $\Delta \mathrm{t}=16 \mathrm{ps}$ pulse of $\sim 1.85 \mathrm{~mJ} / \mathrm{cm}^{2}$ on sample $\mathrm{Gd}_{27} \mathrm{FeCo}$. (b) Calculation of electronic (solid lines) and lattice (dotted lines) temperatures after a $\Delta \mathrm{t}=55 \mathrm{fs}, \mathrm{F}_{\mathrm{c}}=0.8 \mathrm{~mJ} / \mathrm{cm}^{2}$ pulse (red), a $\Delta \mathrm{t}=1 \mathrm{ps}, \mathrm{F}_{\mathrm{c}}=0.9 \mathrm{~mJ} / \mathrm{cm}^{2}$ pulse (blue), and a $\Delta \mathrm{t}=12.5 \mathrm{ps}, \mathrm{F}_{\mathrm{c}}=1.35 \mathrm{~mJ} / \mathrm{cm}^{2}$ pulse (black) according to the threetemperature model. The dashed line indicates $\mathrm{T}_{\mathrm{c}}$. For $\Delta \mathrm{t}=12.5 \mathrm{ps}$ pulses, $\mathrm{T}_{\mathrm{e}}$ gets very close to $\mathrm{T}_{\mathrm{c}}$. Whether $\mathrm{T}_{\mathrm{e}}$ needs to reach $\mathrm{T}_{\mathrm{c}}$ or not is unclear due to the uncertainties $(\sim 20 \%)$ of the critical fluences $\mathrm{F}_{\mathrm{c}}$. Adapted figures with permission from [21]. Copyright 2016 by the American Physical Society.

that electrons need to be heated very fast (with sub-picosecond pulses) in order to meet the required non-equilibrium, high electron temperatures.

\subsection{Picosecond optical pulses for all-optical switching}

In order to test the requirements on electron temperatures, we progressively stretched our optical pulses up to 20 ps to observe if, and when, AOS would be no longer possible [21]. As the pulse duration $\Delta$ t increases, the laser peak intensity drops as $1 / \Delta t$ resulting in a lower peak electron temperature $\mathrm{T}_{\mathrm{e}}$. However, since energy transfer rates depend on temperature differences between heat baths, electrons actually lose less energy when they are cool. The result is a drop of the peak $\mathrm{T}_{\mathrm{e}}$ by a factor of $\sim 3$ when going from $\Delta \mathrm{t}=$ 50 fs to $10 \mathrm{ps}$.

For the measurements in [21], we reset the film with an external magnetic field, irradiated it with single laser pulses and verified the toggle-switching behavior via a magneto-optical Kerr effect (MOKE) microscope. Figure 3a 
shows the measured critical energy threshold for AOS (red triangles) as a function of pulse duration, up to a maximum of $15 \mathrm{ps}$, above which no more AOS was observed in any sample. More strikingly, the required energy increased only by $\sim 50 \%$ as the pulse duration increased by over two orders of magnitude. A limit on the pulse duration was also found, corresponding to when the threshold energy is such that the lattice overcomes $\mathrm{T}_{\mathrm{c}}$ (blue dashed line). Exceeding this threshold results in a loss of magnetic memory and a nucleation of random domains.

Based on these findings, we performed a three temperature model simulation in order to estimate peak temperatures at the recorded energy thresholds and pulse durations [21]. The striking difference in peak electron temperatures between a 55 fs and a 12.5 ps pulse is visible in Fig. 3b (solid lines). Our results proved that, contrary to previous belief, a high peak electron temperature is not required for AOS. Moreover, we note that two other works had already studied the requirements on pulse duration, and had arrived to similar conclusions, in the context of helicity-dependent AOS in GdFeCo [59, 60]. Due to the experimental uncertainties, according to our simulations, it is not clear whether surpassing $T_{c}$ is needed (see solid black line in Fig. 3b).

The speed of the reversal was verified via time-resolved MOKE experiments [21]. Even for long, 10 ps-wide pulses, the reversal (crossing of zero magnetization) happens at around $13 \mathrm{ps,} \mathrm{which} \mathrm{is} \mathrm{still} \mathrm{relatively} \mathrm{fast} \mathrm{com-}$ pared to conventional magnetization reversal dynamics.

The realization that picosecond heating of the electrons is enough for an ultrafast magnetization reversal in GdFeCo opens new possibilities for applications but also raises a new question. Is direct optical irradiation necessary, or can the heat be sourced by an adjacent, hot layer?

\subsection{All-optical switching mediated by electronic transport}

In recent years, a number of works have demonstrated that femtosecond optical pulses can launch heat, spin and/or electronic current pulses that can, themselves, trigger interesting magnetization dynamics in an adjacent magnetic film $[61,62,63,64,65]$. We fabricated a series of samples to test whether a similar experiment generating a pure ultrafast heat pulse would switch a GdFeCo film, in the absence of any direct optical excitation [12].

The samples consisted of $\operatorname{Pt}(6 \mathrm{~nm}) / \mathrm{Au}(\mathrm{t} \mathrm{nm}) / \mathrm{GdFeCo}(10 \mathrm{~nm}) /$ sapphire, as shown in Fig. 4a, and were always excited through the top highlyabsorbing Pt layer [12]. The Au spacer layer, of thickness t, was included to tune the amount of direct optical absorption in the GdFeCo. Via multilayer 

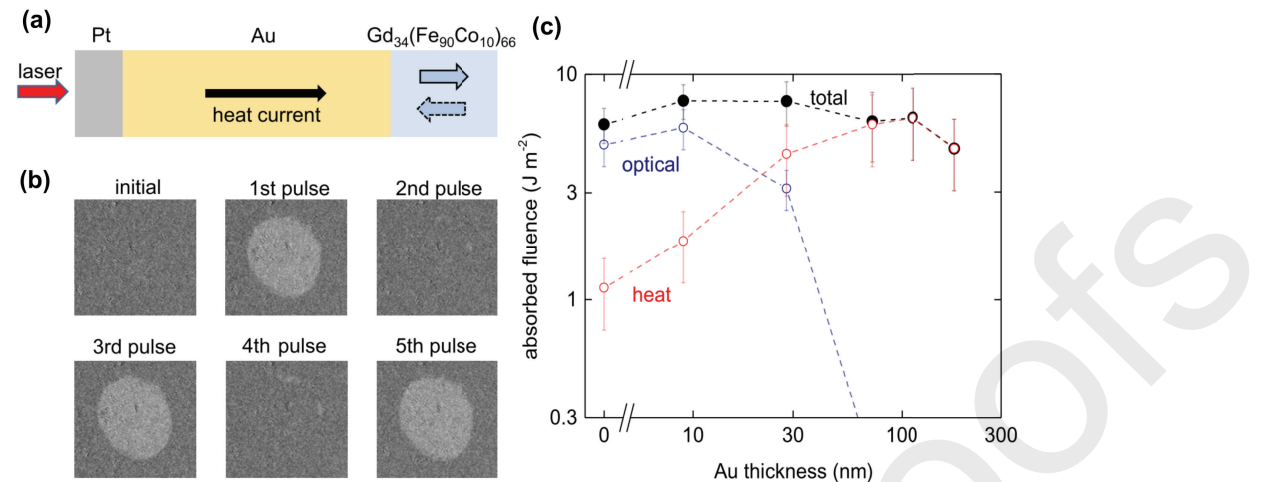

Figure 4: (a) Schematic of experiment. A laser pulse irradiates the Pt surface and deposits energy in the $\mathrm{Pt}$ and $\mathrm{Au}$ electrons. Hot electrons diffuse across the Au layer and heat the GdFeCo. (b) MOKE micrographs of the GdFeCo magnetization in a 6-nm Pt/113-nm $\mathrm{Au} / 10-\mathrm{nm}$ GdFeCo trilayer after the Au surface is successively irradiated with linearly polarized laser pulses. The sample's initial magnetization is down (M-). (c) Estimate of fluence absorbed by $\mathrm{GdFeCo}$ electrons vs. Au film thickness. The red open circles represent the fluence from heat currents via the Au electrons, the blue open circles represent the fluence from direct optical absorption, and the filled black circles represent the total fluence absorbed by the GdFeCo electrons from all sources. Lines are to guide the eye. Adapted figures with permission from [12]. Copyright 2017 by the American Physical Society.

optical calculations, we estimated that for $\mathrm{t}>30 \mathrm{~nm}$ the amount of optical energy absorbed by the GdFeCo layer was negligible.

We irradiated all the films with single 55 fs pulses from an $810 \mathrm{~nm}$ wavelength laser and checked for AOS using a MOKE microscope, by imaging through the transparent substrate. AOS was observed for all Au thicknesses, even for $\mathrm{t}=200 \mathrm{~nm}$, as shown in Fig. $4 \mathrm{~b}$. To achieve switching, however, the required incident energy had to be increased by a factor of $\sim 10$, going from $\mathrm{t}=0$ to $\mathrm{t}=113 \mathrm{~nm}$, since some of the energy was lost to the Pt/Au layers before even reaching the GdFeCo. For each Au thickness, the fraction of the critical optical fluence reaching the GdFeCo layer is plotted in Fig. 4c (blue curve). Above $\mathrm{t}=30 \mathrm{~nm}$, almost no direct optical energy is absorbed by the GdFeCo layer. This necessitates that, for films with thicker Au, the energy driving the switching must be of a different origin: heat transport initiated by the hot $\mathrm{Pt} / \mathrm{Au}$ layers.

Upon optical irradiation of a metal film, electrons are first excited to high energy levels resulting in a non-thermal electron distribution (where Fermi-Dirac statistics don't apply). These electrons will then travel ballis- 
tically until they scatter and lose energy to lower-energy electrons, giving rise to the thermalization of the electron bath. This multitude of scattering events gradually transforms into electron diffusion, which is defined only when the electron population is fully thermalized and temperatures can be defined. Until total electron thermalization has been reached, a so-called super-diffusive transient regime [66] has been proposed to describe the behavior. Although the possible co-existence of various transport mechanisms following irradiation is well accepted, the timescales and length scales involved are not. So how can we tell if the electrons that carry most of the energy to our GdFeCo layer are, in nature, ballistic (high energy, thus similar to an optical absorption) or diffusive (low energy)?

One way to answer to this question is to identify the delay in the arrival of the heat pulse by measuring the (delayed) magnetic dynamics as a function of spacer thickness. If transport is ballistic, the pulse should arrive within a few hundred femtoseconds at most, since electrons travel at the Fermi velocity $(1 \mathrm{~nm} / \mathrm{fs})$. However, our time-resolved MOKE experiments [12] revealed a longer delay $(\sim 1 \mathrm{ps})$, compatible with diffusive energy transport. Nevertheless, the same experiments confirmed that the magnetization reversal was still ultrafast.

A second way to address the question about the nature of the transport is to check whether simple diffusive models predict enough heat currents to allow for switching of GdFeCo. We first performed a calculation [12] that yields a heat current estimation (shown in red in Fig. 4c) that increases as the thickness of $\mathrm{Au}$ is increased. The resulting total absorbed energy (optical + heat transport) in the GdFeCo layer stayed nearly constant at around $5 \mathrm{~J} / \mathrm{m}^{2}$. A constant switching energy threshold is expected if the effective heat pulse duration is not excessively broadened [21]. Finally, we compared the percentage of optical energy absorbed in the $\mathrm{Pt} / \mathrm{Au}$ layers with the percentage our model predicted would reach the GdFeCo layer. Model estimations were based on our time-resolved demagnetization traces for the same samples (see details in ref. [12]). Good agreement between the experimental behavior and our calculations reinforced the idea that most of the energy responsible for the switching of GdFeCo in the $200 \mathrm{~nm} \mathrm{Au} \mathrm{film} \mathrm{is}$ due to thermalized, low-energy, diffusive hot electrons.

In conclusion, our experiments and analyses showed that a pure heat pulse of thermalized electrons is able to reverse GdFeCo [21]. This likely means that any rapid excitation carrying enough energy to quickly raise the electronic temperature to a certain point should suffice to trigger the magnetization 
switching in GdFeCo regardless of the initial electronic distribution. Finally, this result confirms that picosecond electrical pulses can carry the required heat energy and should be able to reverse the magnetic state of a GdFeCo device, thus opening the door to an all-electronic ultrafast magnetic memory.

\section{Electrical Current-Based Ultrafast Devices}

Prior to driving ultrafast magnetization switching electrically, we first consider the differences between optical irradiation and electrical stimulation. In short, optical irradiation of magnetic metals makes electrons hot. Hot electrons drive ultrafast magnetic phenomena in two ways. First, hot electrons drive a complex cascade of electron-electron, electron-phonon, and electron-magnon scattering processes. These scattering processes result in flows of energy and angular momentum between electronic, spin, and vibrational degrees-of-freedom $[1,8,67,68]$. Second, spatial gradients in internal energy drive transport phenomena [69, 70, 71, 55, 72, 73]. Numerous theoretical and experimental studies have credited ultrafast spin-currents as a key driver of ultrafast magnetic phenomena [69, 70, 55, 74].

Optical irradiation is not the only method capable of generating hot electrons in a metal, however. Electrical currents driven at frequencies much lower than those of visible light can also generate a nonequilibrium state. At room temperature, however, where electron-phonon coupling is quite strong [75], large fields are necessary to induce non-equilibrium.

We can estimate the expected nonequilibrium between electrons and phonons $\Delta T_{e p}$ for a given field-strength $E$ by equating the rate of Joule heating to energy transfer between the lattice, $g_{e p} \Delta T_{e p} \approx \sigma E^{2}$. Here, $g_{e p}$ is the electron-phonon energy transfer coefficient, and $\sigma$ is the electrical conductivity. Our study of all optical switching of GdFeCo with picosecond optical pulses suggests that ultrafast switching requires heating sufficient to drive electrons to temperatures $\sim 100 \mathrm{~K}$ higher than the lattice [21], see Fig. 3b. Typical values for electrical conductivity and electron-phonon coupling in a magnetic thin film are $\sigma \approx 10^{6} \mathrm{~S} / \mathrm{m}$ and $g_{e p} \approx 10^{18} \mathrm{Wm}^{-3}$. Therefore, a simple back-of-the-envelope calculation suggests a field strength on the order of $10^{7} \mathrm{~V} / \mathrm{m}$ applied for ten picoseconds will drive a magnetic metal into a nonequilibrium state comparable to the one caused by irradiation by a ten-picosecond optical pulse. Such field strengths are achievable in microelectronic devices, where small dimensions make large field strength possible. 
To demonstrate the ability of picosecond electrical pulses to drive ultrafast dynamics in GdFeCo, we fabricated thin magnetic metal films into coplanar strip lines with integrated photoconductive Auston switches [13, 14]. Following irradiation with an ultrafast laser pulse, the photoconductive Auston switches generate electrical pulses with durations ranging from 1-10 picoseconds. The pulse duration depends on a number of factors, including the semiconductor carrier recombination rate, the design of the Auston switch, the electrical capacitance of the switch, and the duration of the laser pulse. In our fabricated structures, the strip line is tapered down from a $50 \mu \mathrm{m}$ width to $5 \mu \mathrm{m}$ width to increase the field magnitude. A $4 \mu \mathrm{m}$ long section of the $5 \mu \mathrm{m}$ wide strip-line is made from a nanoscale magnetic film. When the picosecond electrical pulses pass through the magnetic film section of the strip-line, high electrical current densities result in hundreds of pico-Joules of energy being deposited into the electrons of the magnetic metal $[13,14]$.

In Fig. 5, we show ultrafast switching of a $\mathrm{Ta}(5 \mathrm{~nm}) / \mathrm{Gd}_{30} \mathrm{Fe}_{63} \mathrm{Co}_{7}(20$ $\mathrm{nm}) / \mathrm{Pt}(5 \mathrm{~nm})$ film in response to 9 ps electrical pulses, all of the same current polarity. The GdFeCo film possesses perpendicular magnetic anisotropy, a coercivity of 80 Oe, and a compensation temperature of $\sim 270 \mathrm{~K}$. Figure 5 shows MOKE micrographs of the GdFeCo in response to a sequence of 9-ps electrical pulses. After each electrical pulse, the magnetization toggle switches to the opposite direction as previously observed with optical pulses.

To determine the speed of the switching, we performed time-resolved MOKE measurements of the dynamics. After irradiating the Auston switch with an optical pump pulse, an electrical current pulse is generated from the switch and propagated down the transmission line. We then measure the MOKE signal from the GdFeCo film with a time-delayed optical probe pulse, see Fig. 5. Reversal of the magnetization direction occurs on a 10 ps timescale (defined by the zero-point crossing of the magnetization), similar to the duration of the electrical pulse. Following reversal, the FeCo magnetization recovers rapidly toward a new equilibrium state in the opposite direction. It reaches $70 \%$ of saturation within just 30 ps. As heat diffuses out of the metal film, the magnetization reaches saturation, i.e. complete reversal, on a 1 ns timescale.

In addition to ultrafast heating, the electrical pulses will induce dynamics via an oersted field and spin-orbit-torques from the Pt and Ta layers. The associated oersted field is on the order of $\sim 70 \mathrm{mT}$, much too small to cause switching within 10 ps. Similarly, the spin-orbit torques produced are also too small to cause ultrafast switching. Macro-spin calculations based on the 
(a)

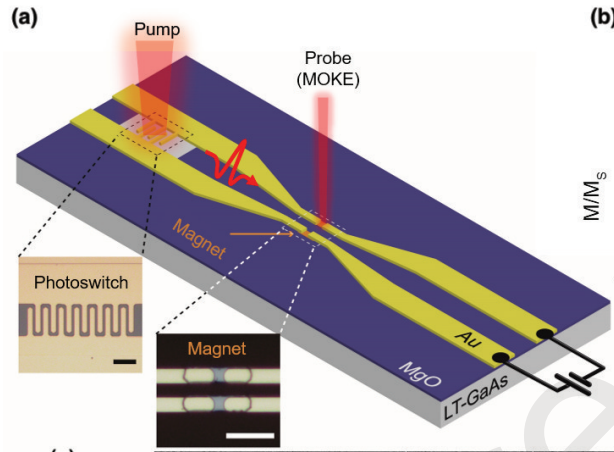

(b)

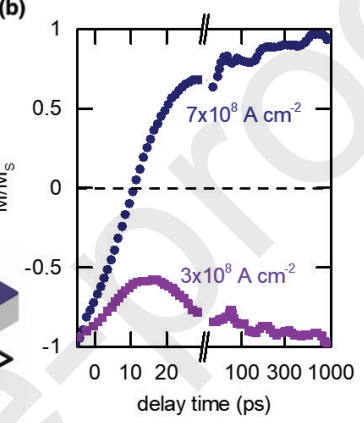

(c)

Electrical
Pulses

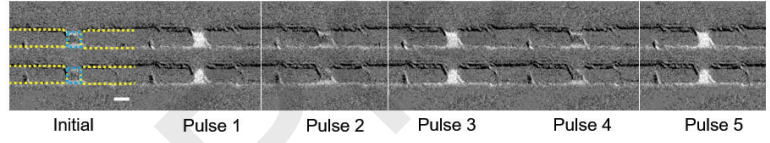

Figure 5: Ultrafast switching of GdFeCo with ten picosecond electrical pulses. (a) Schematic of the electrical switching experiment. The photoswitch is illuminated with laser pulses while being biased with a dc source. (b) Magnetization dynamics of GdFeCo is monitored with time-resolved MOKE. With enough electrical pulse amplitude, the GdFeCo magnetization switches in 10 ps. (c) MOKE images of the GdFeCo CPS section after sequential 9-ps electrical pulse excitation. After each electrical pulse, the magnetization of GdFeCo toggles. Yellow and blue dashed lines indicate gold CPS and GdFeCo sections, respectively. Scale bars, $5 \mathrm{~mm}$. (c) Time-resolved dynamics after excitation with different current densities. Adapted from [14]. (C) The Authors, some rights reserved; exclusive licensee American Association for the Advancement of Science. Distributed under a Creative Commons Attribution NonCommercial License 4.0 (CC BY-NC) http://creativecommons.org/licenses/by-nc/4.0/ 
Landau-Lifshitz-Gilbert equation suggest the change in the out-of-plane magnetization from spin-orbit torque will be less than $10 \%$ for the field strengths of $E \sim 10^{7} \mathrm{~V} / \mathrm{m}$ in our experiments [14].

Our experiments demonstrate that picosecond electrical pulses are effective at generating hot electrons and driving ultrafast switching. However, we do observe differences in the dynamics that result from electrical vs. optical pulses. For example, in similar experiments that we carried out on $\mathrm{Co} / \mathrm{Pt}$ multilayers [13], we observed significantly different ultrafast demagnetization dynamics of the Co in response to electrical heating as opposed to optical heating, see Fig. 6. Similar experiments by Bonetti et al. with free-space $\mathrm{THz}$ irradiation also suggest the dynamics induced by $\mathrm{THz}$ fields differ from optical irradiation [76]. Developing a detailed understanding of the origins of these differences requires further study. However, our work to date suggests that differences in the initial distribution of hot electrons plays an important role [13]. Electrical heating excites a large population of electrons within tens of meV of the Fermi level [13], while optical irradiation excites a smaller number of electrons with eV-scale energies [77, 78, 79]; both are pictorially illustrated in Fig. 6. Nevertheless, our work establishes that regardless of the source from which these hot electrons are produced, whether by direct optical irradiation, hot electron diffusion, electrical current pulse generation, or $\mathrm{THz}$ irradiation, the final result of ultrafast demagnetization or ultrafast switching of magnetic materials can still be the same.

\section{Towards Realistic Applications}

The ability to trigger ultrafast magnetization switching using electrical current pulses can have tremendous impact on integrated electronic and spintronic devices. The previous section described how we experimentally demonstrated switching by picosecond electrical pulses that we generated optically from optoelectronic switches. However, this type of current pulse generation is not necessary. Instead, picosecond current pulses can be easily generated by today's complementary metal-oxide semicondutor (CMOS) transistor nodes [80]. Thus, we can now conceive of integrating ultrafast spintronics given the possibility of driving ultrafast electrical switching of magnetization using conventional CMOS technology.

Before complete integration of ultrafast spintronics may be possible, a few challenges remain to be overcome. First, as discussed previously, an integrated device would require an electrical readout. Therefore, in addition to 

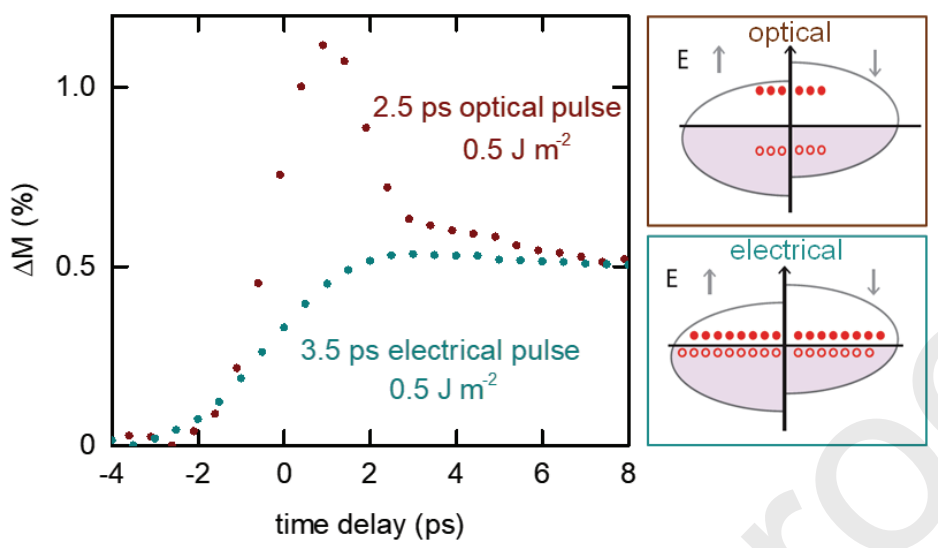

Figure 6: Time-resolved MOKE measurements of ultrafast demagnetization of a $\mathrm{Co} / \mathrm{Pt}$ multilayer film. Following irradiation with a $2.5 \mathrm{ps}$ optical pulse the magnetization decreases by $1 \%$ and then recovers quickly to a new equilibrium at an elevated temperature with a magnetization $0.5 \%$ lower than that before the excitation. Alternatively, the same amount of energy deposited electrically results in a much smaller peak demagnetization. We credit the different dynamics to differences in the number and average energy of initially excited distribution, illustrated on the right. Adapted from data in Ref. [13]

triggering the magnetization switching (the "write" operation) electrically, an ultrafast device could use a magnetic tunnel junction (MTJ) to "read" the magnetization state in the form of a resistance. However, MTJs made with ferrimagnetic GdFeCo have poor tunneling magnetoresistance compared to tunnel junctions made from more conventional ferromagnetic materials [56]. Second, energy and density requirements demand that any integrated ultrafast devices be scaled down to nanometer sizes. Only at these dimensions can the switching energy be reasonably sourced by an integrated circuit. Smaller devices also allow more of them to be incorporated into a given area. Thus, in an effort to integrate ultrafast spintronics, we must determine whether the physics of ultrafast switching scales down to nanometer dimensions. Finally, complete integration will likely require the incorporation of rare-earth transition-metal alloys into devices with a more complex material stack that interfaces with transistor-level circuitry. In this section, we detail experiments that address the first two challenges. We show that ultrafast switching of GdFeCo will also drive the reversal of an adjacent exchange coupled ferromagnetic layer. We then show ultrafast switching of patterned GdCo dots with nanometer-scale diameters. 


\subsection{Ferromagnet single shot HI-AOS}

A potential strategy for overcoming the low tunneling magnetoresistance of $\mathrm{GdFeCo}$ tunnel junctions is to couple it to a ferromagenetic layer, while maintaining the ultrafast switching of the two layers together. Tunnel junctions with ferromagnetic layers as electrodes, usually $\mathrm{Fe}$, Co, or a $\mathrm{CoFeB}$ alloy, on both sides of the barrier can possess tunneling magnetoresistance ratios orders of magnitude higher than that observed for a GdFeCo junction $[28,29,30,56]$. To explore this avenue, in [81], we exchange coupled a ferromagnet to $\mathrm{GdFeCo}$ and found that the GdFeCo largely extended its ultrafast switching capabilities to the ferromagnet.

In this experiment, $\mathrm{Co} / \mathrm{Pt}$ served as the ferromagnetic material, where an additional $\mathrm{Pt}$ spacer of thickness d between the ferrimagnetic GdFeCo and the ferromagnetic $\mathrm{Co} / \mathrm{Pt}$ defined whether the exchange coupling was ferromagnetic or antiferromagnetic in nature based on RKKY coupling [82, $83,84,85]$. The two types of couplings were characterized using depthsensitive MOKE hysteresis measurements [86]. For $\mathrm{d}=1.5$ to $3 \mathrm{~nm}$, the coupling was ferromagnetic and the hysteresis exhibited a typical squareloop shape. For $\mathrm{d}=4$ and $5 \mathrm{~nm}$, samples exhibited four remanent states, where minor hysteresis loops unveiled an antiferromagnetic coupling.

Single shot experiments were done on films with the structure Ta (3 $\mathrm{nm}) / \mathrm{GdFeCo}(20 \mathrm{~nm}) / \mathrm{Co}(0.4 \mathrm{~nm}) / \mathrm{Pt}(\mathrm{d}) / \mathrm{Co}(0.6 \mathrm{~nm}) / \mathrm{Pt}(3 \mathrm{~nm})$. Noticeable differences in the switching regions were observed for the two types of coupling, as seen in Fig. 7. Ferromagnetically-coupled samples show a single switching region corresponding to where the laser intensity is above the critical fluence needed to switch both the GdFeCo and $\mathrm{Co} / \mathrm{Pt}$ together. Subsequent laser shots result in reproducible toggle switching of the magnetization, as expected of GdFeCo. Antiferromagnetically-coupled samples, on the other hand, are characterized by a more complex switching behavior. The sample is initialized with a very strong field such that both layers are aligned parallel (against the film's natural desire to be aligned antiparallel). As in other laser shot experiments, the outer region of the sample beyond the laser spot remains unchanged during the experiment. However, the region stimulated by the laser pulse is divided into two sections. In the inner section, experiencing the highest intensity from the Gaussian pulse, the excitation is enough to completely switch the magnetization of the GdFeCo layer such that it aligns antiparallel to the $\mathrm{Co} / \mathrm{Pt}$. Although the $\mathrm{Co} / \mathrm{Pt}$ is significantly heated in the process, its magnetization remains unchanged, because this new anti-aligned configuration is already the lowest energy state. 

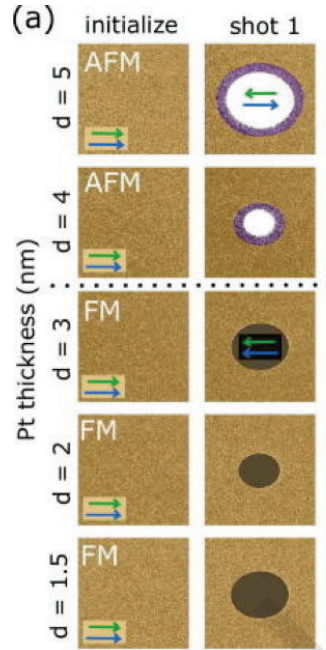
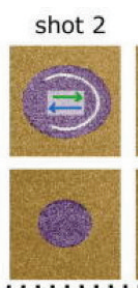

$\ldots \ldots$
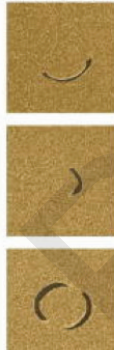
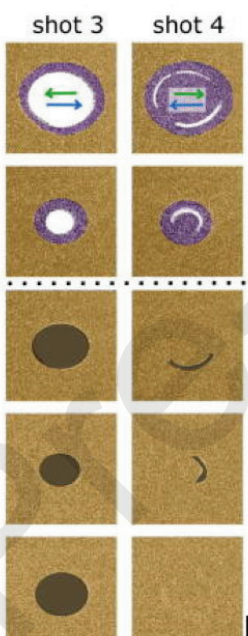

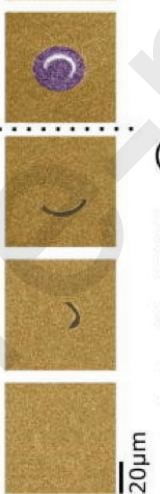

(b)

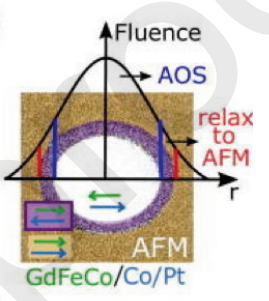

(c)

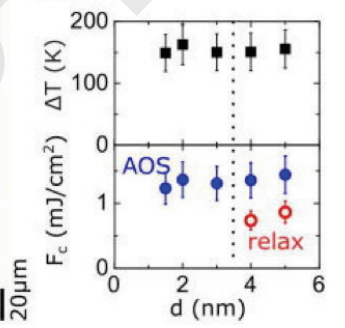

Figure 7: Single shot all-optical switching of both Co/Pt and GdFeCo layers. (a) Digitally re-colored MOKE images of a sequence of $\mathrm{AOS}$ events on the $\mathrm{GdFeCo} / \mathrm{Co} / \mathrm{Pt}(\mathrm{d} \mathrm{nm}) / \mathrm{Co}$ stack series. Green and blue arrows represent $\mathrm{GdFeCo}$ and $\mathrm{Co} / \mathrm{Pt}$ magnetizations, respectively. (b) Laser intensity profile and resulting domain configuration on film $\mathrm{d}=5 \mathrm{~nm}$ after the first laser shot. (c) Absorbed critical fluence $\mathrm{F}_{\mathrm{c}}$ for AOS (blue filled circles), $\mathrm{F}_{\mathrm{c}}$ for relaxation of parallel states following the AFM coupling (red empty circles, and estimated peak lattice temperature rises $\Delta \mathrm{T}$ (black squares) as a function of the $\mathrm{Pt}$ spacer thickness d. Reprinted from [81], with the permission of AIP Publishing. 
(a)
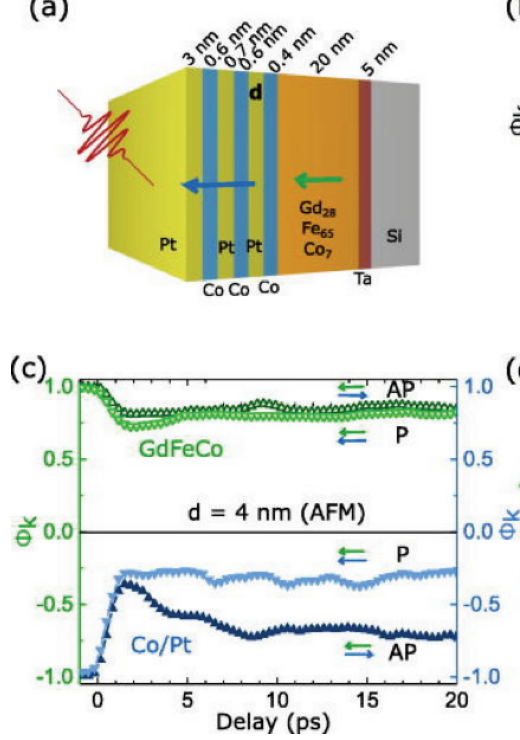

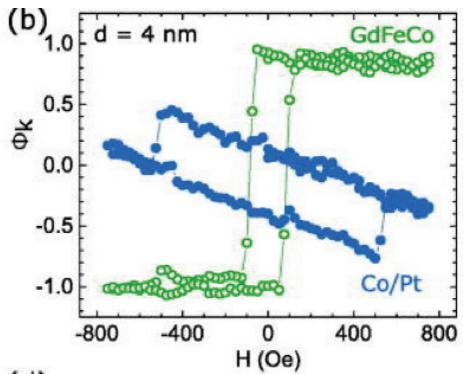

(d)

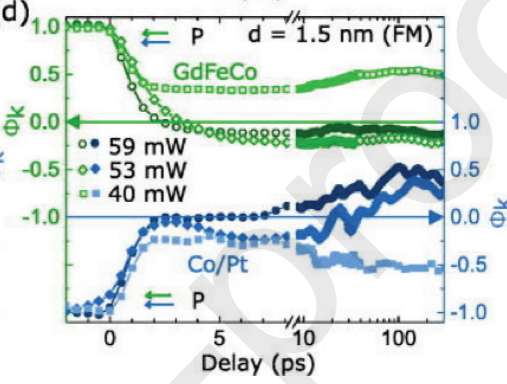

Figure 8: Time-resolved depth-sensitive magneto-optical measurements of laser induced dynamics. (a) $\mathrm{GdFeCo} / \mathrm{Co} / \mathrm{Pt}(\mathrm{d} \mathrm{nm}) /[\mathrm{Co} / \mathrm{Pt}] 2$ stack series with $\mathrm{Pt}$ spacer of thickness d. (b) Depth-sensitive MOKE magnetic hysteresis loops on sample d = $4 \mathrm{~nm}$. (c) Depthsensitive time-resolved demagnetization curves for antiparallel (AP) or parallel (P) initial states of the stack $\mathrm{d}=4 \mathrm{~nm}$. Green and blue arrows represent GdFeCo and $\mathrm{Co} / \mathrm{Pt}$ magnetizations, respectively. (d) Depth-sensitive demagnetization and AOS experiments at various fluences on sample $d=1.5 \mathrm{~nm}$. Reprinted from [81], with the permission of AIP Publishing.

In the ring between the inner and outer regions, though, the laser intensity is insufficient to switch the GdFeCo but sufficient to heat the $\mathrm{Co} / \mathrm{Pt}$ enough to reverse its magnetization relative to the GdFeCo layer and relax the system to the lowest energy state. Equally complex spatial behavior is also observed in the recent work by Iihama et al. [16].

Time-resolved switching experiments were done on an almost identical material stack of Ta $(5 \mathrm{~nm}) / \mathrm{GdFeCo}(20 \mathrm{~nm}) / \mathrm{Co}(0.4 \mathrm{~nm}) / \mathrm{Pt}(\mathrm{d}) / \mathrm{Co}(0.6$ $\mathrm{nm}) / \mathrm{Pt}(3 \mathrm{~nm})$ where an integrated Au coil was patterned on top to reset the magnet between pulses without influencing the magnetization dynamics. Depth-sensitive MOKE once more allowed for the TR-MOKE to measure only a single magnetic layer at a time. For the antiferromagnetically-coupled sample, only time-resolved demagnetization experiments could be done because the maximum field produced by the patterned coil was smaller than the saturation field of the material ( $\sim 500$ Oe). The sample was initialized 
to either the parallel (P) or antiparallel (AP) state and then excited by the laser pulse. As shown, in Fig. 8c, the initial demagnetization in the first 1-2 ps is the same for both samples. Afterwards, the sample initialized as AP settles back to its initial state much faster than the one initialized to P. This is believed to be because the sample prefers to settle to its lowest energy AP state. In the ferromagnetically-coupled sample, the TR-MOKE behavior in Fig. 8d is much more intuitive. At low laser powers, only demagnetization of both the GdFeCo and $\mathrm{Co} / \mathrm{Pt}$ layers is observed. Eventually, at higher powers, the GdFeCo switches (crosses zero) within $2 \mathrm{ps}$, and pulls the $\mathrm{Co} / \mathrm{Pt}$ along, making it reverse at around 7 ps.

These results imply that the exchange coupling is sufficient to couple a ferromagnet with GdFeCo and push the ferromagnet to also switch ultrafast within picoseconds. Future work can take advantage of this by constructing magnetic tunnel junctions where the free layer is exchange-coupled to a GdFeCo layer that would be triggered to induce ultrafast switching of magnetic memory technology.

\subsection{Ultrafast switching of nanoscale magnetic dots}

Another aspect of paving the way towards ultrafast spintronics integration is determining the phenomenon's behavior and compatibility with nanometer length-scale patterning. Until now, the roles of electrons, spins, and phonons in HI-AOS are not yet completely understood. Furthermore, in nanometer-scale devices, quantum mechanical interactions are expected to be much more pronounced due to the structural confinement. Therefore, it is unclear how dimensional down-scaling would influence the ultrafast behavior of GdFeCo. Previous attempts to confine the magnetization reversal to a nanoscale region within larger $\mathrm{TbFeCo}$ alloy films found it difficult to precisely control the switching location and behavior as a result of inhomogeneity in the local anisotropy [87, 88]. Micro- and nanometer scale patterning of GdFeCo was attempted by Le Guyader et al. [89, 90], but the magnetization was often complicated by the low perpendicular magnetic anisotropy (PMA) of GdFeCo, especially at diameters below $1 \mu \mathrm{m}$ [90]. Our work in [91] aimed to reduce the dimensions of the patterns to the nanoscale while still preserving both PMA and ultrafast HI-AOS.

In our initial attempts to pattern a $\mathrm{Ta}(5 \mathrm{~nm}) / \mathrm{Gd}_{27} \mathrm{Fe}_{66} \mathrm{Co}_{7}(10 \mathrm{~nm}) / \mathrm{Pt}$ (5 nm) film, we observed similar loss of PMA for patterned sizes smaller than $1 \mu \mathrm{m}$. However, patterning of a GdCo-alloy instead yielded PMA down to the smallest nanoscale patterns detectable $(200 \mathrm{~nm})$ with laser MOKE. 


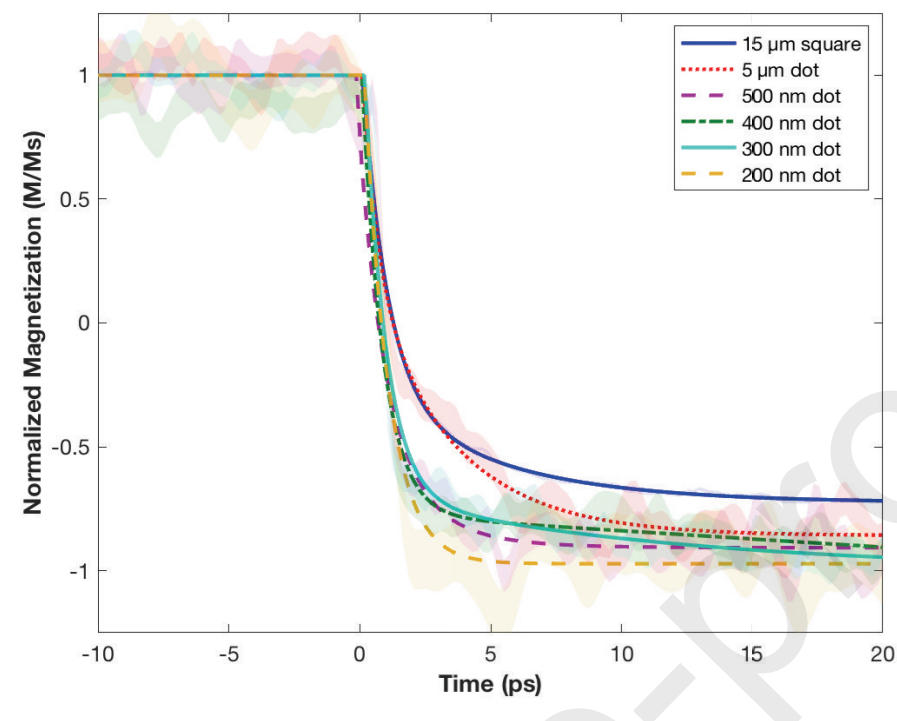

Figure 9: Time resolved magnetization switching behavior of micro and nanoscale dot patterns of a-Gd-Co, all taken at an incident fluence of $7 \mathrm{~mJ} / \mathrm{cm}^{2}$. The normalized experimental data is represented by the shaded regions with width of one standard deviation. The curve fits summarize the behavior for each size. A clear trend can be seen where smaller patterns settle to the reversed magnetization state at faster rates than larger dots. Adapted from [91], with the permission of AIP Publishing.

Therefore, this latter material, consisting of the material stack Ta $(3 \mathrm{~nm}) / \mathrm{Pt}$ $(3 \mathrm{~nm}) / \mathrm{Gd}_{30} \mathrm{Co}_{70}(10 \mathrm{~nm}) / \mathrm{Pt}(3 \mathrm{~nm})$ was patterned into dot arrays and utilized for HI-AOS experiments.

Arrays of patterned magnetic dots with diameter $\mathrm{d}$ and spacing $2 \mathrm{~d}$ were made to fill areas of size $25 \mu \mathrm{m} \times 25 \mu \mathrm{m}$. Each array of uniform dot size was then measured by laser MOKE using a Ti-Sapphire laser with wavelength $810 \mathrm{~nm}$ and pulse width of $70 \mathrm{fs}$. Single shot switching was observed down to the smallest detectable size of $200 \mathrm{~nm}$, despite the noisy measurement at such small dimensions. Prior to these results, a similar material only in the form of synthetic ferrimagnets made of $\mathrm{Pt} / \mathrm{Co} / \mathrm{Gd}$ stacks demonstrated HIAOS [57], whereas alloys of composition $\mathrm{Gd}_{17} \mathrm{Co}_{83}$ exhibited only ultrafast demagnetization [92]. However, the present results indicate that GdCo alloys with slightly higher Gd concentration than the latter can toggle switch like GdFeCo alloys in response to femtosecond laser excitation.

Time-resolved MOKE experiments were utilized to study the time-domain 


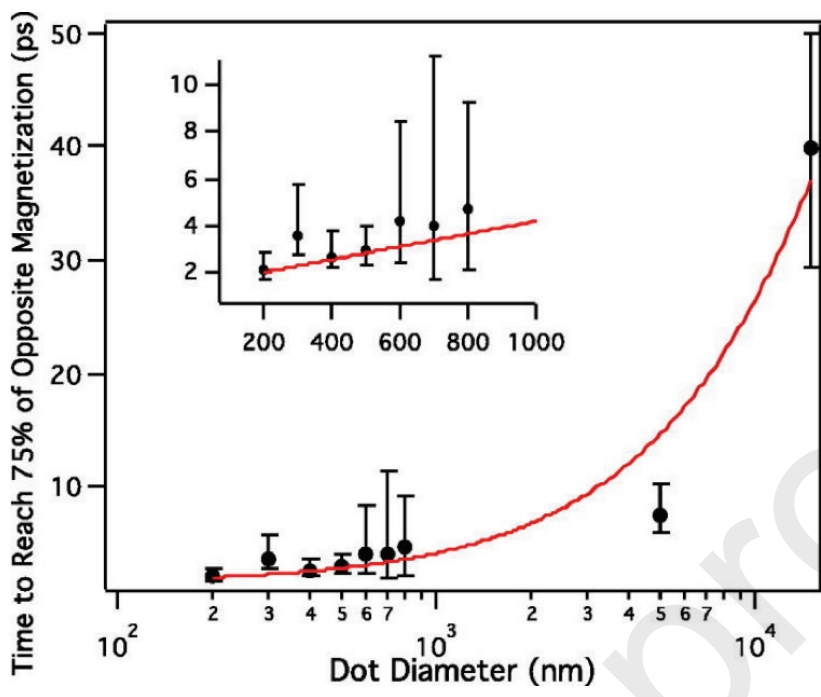

Figure 10: The time required for the magnetization to cross a $75 \%$ reversal threshold based on fitting of the TR-MOKE data for different dot diameters. The $75 \%$ reversal threshold can be used to classify switching in digital electronics. The red curve is a guide to the eye for the data as a function of size. The inset is an expanded view of the smaller nanodot data. Asymmetric error bars arise from the uncertainty in the $75 \%$ reversal time, particularly given the long tail in the remagnetization TR-MOKE data as compared to the much more rapid demagnetization. Reprinted from [91], with the permission of AIP Publishing.

switching behavior as a function of pattern size. Interestingly, a trend could be seen from the data in Fig. 9 where dots of smaller diameters settled to the reversed magnetization direction at a faster rate. Increased levels of noise in the results for smaller patterns are assumed to be a result of non-uniform light absorption with obliquely-incident light [93] as well as edge effects, drift in the laser power and position, and spatial interference patterns arising from the light's interaction with the nanodot array. Nevertheless, the trend as a function of dot size is clear in Fig. 9 and is quantified in Fig. 10 in terms of the time each pattern size takes to reach a $75 \%$ reversal threshold useful for determining the digital state in integrated electronics. What can be seen is that the state of a large $15 \mu \mathrm{m}$ pattern takes $40 \mathrm{ps}$ to be written to the opposite state (assuming a 75\% threshold), as compared to a $200 \mathrm{~nm}$ dot, which can be written in just 2 ps. Such rapid write times present tremendous potential for ultrafast spintronics integration.

To understand the experimental trend observed in Fig. 10, we pro- 
pose considering the rates of relevant electron-phonon and spin-lattice energy transfer processes in the first few picoseconds after the laser stimulus [91]. In large continuous films, electron-phonon equilibration occurs within $\sim 1 \mathrm{ps}$ [94]. For patterned dots with smaller lateral dimensions, the electron-phonon coupling is known to increase [95, 96, 97] due to increased surface scattering, therefore yielding shorter spin flip scattering times. It follows that increased spin flip processes could mean increased rates for the magnetization dynamics, which would be of particular importance to the rare-earth sublattice since its dynamics are significantly slower than those of the transition metal sublattice. In the end, this yields an overall faster cooling and recovery of the magnetization, resulting in an overall faster switching speed for nanoscale devices. This faster switching behavior can ultimately allow nanoscale ultrafast spintronic memories and other devices to be written by short picosecond excitations then subsequently equilibrate their magnetization on the same timescale.

\section{Conclusions}

The future of the field of ultrafast spintronics promises significantly faster control of device magnetization for a similar cost in energy to current spintronic technologies. Directly reversing the magnetization direction with current via spin-transfer torque (STT) is much more energy efficient than switching via current-induced magnetic field [98]. However, fast STT switching, on the order of a few hundred picoseconds to nanoseconds [99, 36, 35, 42], requires a very high critical current level, often near the breakdown of the tunnel barrier in a magnetic tunnel junction. Therefore, driving STT devices at high speeds gradually degrades the barrier and consumes significant dynamic energy during write operations. Spin-orbit torque (SOT) switching avoids driving current through and degrading the barrier [100]. Although, as with STT-switching, SOT techniques struggle to maintain low dynamic energy for fast precessional switching speeds, since the critical current required for switching increases significantly as the pulse width is reduced [42]. (However, we note that we very recently demonstrated energy efficient SOT switching with single picosecond electrical pulses [101].) Given the challenges with current-induced switching, voltage-controlled/magnetoelectric switching has risen as an alternative or, perhaps, as a supplement to STT and SOT to reduce the critical current required for switching [102, 46, 103, 104, 47, 105]. The switching speed, however, is still limited to the slower of either the 
magnetoelectric change or the precessional reversal of the magnetic moment.

In this review, we have outlined the potential of ultrafast magnetism and demonstrated progress towards this new regime of spintronics in which excitation of ultrafast magnetization dynamics can be initiated energy-efficiently by picosecond pure charge current pulses. We have also presented related developments towards device integration of ultrafast spintronics through such achievements as ultrafast, single-shot switching of ferromagnets and ultrafast switching of magnetic nanostructures. These advancements may resolve some of the existing challenges in field of spintronics and, in turn, open up many new opportunities for useful applications of ultrafast magnetism in integrated electronics, without the need for femtosecond lasers.

As this article is appearing in a special issue in honor (in part) of the late Professor Jean-Yves Bigot, we wish to acknowledge his interest in and contributions to the work reviewed here. As a pioneer of the field of "femtomagnetism" [1], although primarily focused on the fascinating fundamental physics problems revealed by the experiments, Professor Bigot recognized early on the potential of this field for application to increasing data processing speed in information systems. This was already mentioned in the original 1996 paper [1]. In a 2002 review [106], the point was re-emphasized, and again in a more recent 2013 review of ultrafast magnetization dynamics in nanostructures [107]. During the course of the research work summarized in this paper, over the past $\sim 6$ years, author J.B. has had the pleasure of many fruitful discussions about it with Professor Bigot. Bigot made many important and useful suggestions and expressed much support and encouragement. His insights and input will be sorely missed, and we wish to dedicate this paper as well as future endeavors in the field to his memory.

\section{Acknowledgements}

This work was primarily funded by the U.S. Department of Energy, Office of Science, Office of Basic Energy Sciences, Materials Sciences and Engineering Division under Contract No. DE-AC02-05-CH11231 within the Nonequilibrium Magnetic Materials Program (MSMAG). Additional support was provided by the Center for Energy Efficient Electronics Science under the National Science Foundation award number 0939514. Author R. B. W. was supported by the U.S. Army Research Laboratory and the U.S. Army Research Office under contract/grant number W911NF-18-1-0364. Part of the fabrication was performed at the Marvell Nanofabrication Laboratory at 
the University of California Berkeley while part of this work was performed at the Stanford Nano Shared Facilities (SNSF)/Stanford Nanofabrication Facility (SNF), supported by the National Science Foundation under award ECCS-1542152.

[1] E. Beaurepaire, J.-C. Merle, A. Daunois, J.-Y. Bigot, Ultrafast Spin Dynamics in Ferromagnetic Nickel, Physical Review Letters 76 (22) (1996) 4250-4253. doi:https://doi.org/10.1103/PhysRevLett.76.4250.

[2] J. C. Slonczewski, Current-driven excitation of magnetic multilayers, Journal of Magnetism and Magnetic Materials 159 (96) (1996) 1-7.

[3] L. Berger, Emission of spin waves by a magnetic multilayer traversed by a current, Physical Review B 54 (13) (1996) 9353-9358. arXiv:1102.5325, doi:10.1103/PhysRevB.54.9353.

[4] C. D. Stanciu, F. Hansteen, A. V. Kimel, A. Kirilyuk, A. Tsukamoto, A. Itoh, T. Rasing, All-optical magnetic recording with circularly polarized light, Physical Review Letters 99 (4) (2007) 1-4. doi:10.1103/PhysRevLett.99.047601.

[5] J. Hohlfeld, C. D. Stanciu, A. Rebei, Athermal all-optical femtosecond magnetization reversal in GdFeCo, Applied Physics Letters 94 (15). arXiv:0902.3800, doi:10.1063/1.3119313.

[6] M. S. El Hadri, P. Pirro, C.-H. Lambert, S. Petit-Watelot, Y. Quessab, M. Hehn, F. Montaigne, G. Malinowski, S. Mangin, Two types of alloptical magnetization switching mechanisms using femtosecond laser pulses, Physical Review B 94 (6) (2016) 064412. arXiv:1602.08525, doi:10.1103/PhysRevB.94.064412.

[7] C. D. Stanciu, A. Tsukamoto, A. V. Kimel, F. Hansteen, A. Kirilyuk, A. Itoh, T. Rasing, Subpicosecond magnetization reversal across ferrimagnetic compensation points, Physical Review Letters 99 (21) (2007) 14-17. doi:10.1103/PhysRevLett.99.217204.

[8] B. Koopmans, G. Malinowski, F. Dalla Longa, D. Steiauf, M. Fähnle, T. Roth, M. Cinchetti, M. Aeschlimann, Explaining the paradoxical diversity of ultrafast laser-induced demagnetization, Nature Materials 9 (3) (2010) 259-265. arXiv:1301.3573, doi:10.1038/nmat2593. 
[9] I. Radu, K. Vahaplar, C. Stamm, T. Kachel, N. Pontius, H. A. Dürr, T. A. Ostler, J. Barker, R. F. Evans, R. W. Chantrell, A. Tsukamoto, A. Itoh, A. Kirilyuk, T. Rasing, A. V. Kimel, Transient ferromagneticlike state mediating ultrafast reversal of antiferromagnetically coupled spins, Nature 472 (7342) (2011) 205-209. doi:10.1038/nature09901.

[10] T. A. Ostler, J. Barker, R. F. Evans, R. W. Chantrell, U. Atxitia, O. Chubykalo-Fesenko, S. El Moussaoui, L. Le Guyader, E. Mengotti, L. J. Heyderman, F. Nolting, A. Tsukamoto, A. Itoh, D. Afanasiev, B. A. Ivanov, A. M. Kalashnikova, K. Vahaplar, J. Mentink, A. Kirilyuk, T. Rasing, A. V. Kimel, Ultrafast heating as a sufficient stimulus for magnetization reversal in a ferrimagnet, Nature Communications 3 (2012) 666. arXiv:arXiv:1011.1669v3, doi:10.1038/ncomms1666.

[11] A. R. Khorsand, M. Savoini, A. Kirilyuk, A. V. Kimel, A. Tsukamoto, A. Itoh, T. Rasing, Role of magnetic circular dichroism in all-optical magnetic recording, Physical Review Letters 108 (12) (2012) 1-5. doi:10.1103/PhysRevLett.108.127205.

[12] R. B. Wilson, J. Gorchon, Y. Yang, C. H. Lambert, S. Salahuddin, J. Bokor, Ultrafast magnetic switching of GdFeCo with electronic heat currents, Physical Review B 95 (18) (2017) 1-5. doi:10.1103/PhysRevB.95.180409.

[13] R. B. Wilson, Y. Yang, J. Gorchon, C. H. Lambert, S. Salahuddin, J. Bokor, Electric current induced ultrafast demagnetization, Physical Review B 96 (4) (2017) 1-9. doi:10.1103/PhysRevB.96.045105.

[14] Y. Yang, R. B. Wilson, J. Gorchon, C. H. Lambert, S. Salahuddin, J. Bokor, Ultrafast magnetization reversal by picosecond electrical pulses, Science Advances 3 (11). arXiv:1609.06392, doi:10.1126/sciadv.1603117.

[15] Y. Xu, M. Deb, G. Malinowski, M. Hehn, W. Zhao, S. Mangin, Ultrafast Magnetization Manipulation Using Single Femtosecond Light and Hot-Electron Pulses, Advanced Materials 29 (42) (2017) 1-6. doi:10.1002/adma.201703474.

[16] S. Iihama, Y. Xu, M. Deb, G. Malinowski, M. Hehn, J. Gorchon, E. E. Fullerton, S. Mangin, Single-shot multi-level all-optical magnetization 
switching mediated by Spin transport, Advanced Materials 30 (51). doi:10.1002/adma.201804004.

[17] M. L. Lalieu, R. Lavrijsen, B. Koopmans, Integrating all-optical switching with spintronics, Nature Communications 10 (1) (2019) 1-6. doi:10.1038/s41467-018-08062-4.

[18] T. Kampfrath, M. Battiato, P. Maldonado, G. Eilers, J. Nötzold, S. Mährlein, V. Zbarsky, F. Freimuth, Y. Mokrousov, S. Blügel, M. Wolf, I. Radu, P. M. Oppeneer, M. Münzenberg, Terahertz spin current pulses controlled by magnetic heterostructures, Nature Nanotechnology 8 (4) (2013) 256-260. arXiv:1210.5372, doi:10.1038/nnano.2013.43.

[19] A. V. Kimel, M. Li, Writing magnetic memory with ultrashort light pulses, Nature Reviews Materials 4 (3) (2019) 189-200. doi:10.1038/s41578-019-0086-3.

[20] D. Sander, S. O. Valenzuela, D. Makarov, C. H. Marrows, E. E. Fullerton, P. Fischer, J. McCord, P. Vavassori, S. Mangin, P. Pirro, B. Hillebrands, A. D. Kent, T. Jungwirth, O. Gutfleisch, C. G. Kim, A. Berger, The 2017 Magnetism Roadmap, Journal of Physics D: Applied Physics 47 (33). doi:10.1088/0022-3727/47/33/333001.

[21] J. Gorchon, R. B. Wilson, Y. Yang, A. Pattabi, J. Y. Chen, L. He, J. P. Wang, M. Li, J. Bokor, Role of electron and phonon temperatures in the helicity-independent all-optical switching of GdFeCo, Physical Review B 94 (18) (2016) 19-24. doi:10.1103/PhysRevB.94.184406.

[22] M. Baibich, J. Broto, Giant Magnetosresistance of (001)Fe/(001)Cr Magnetic Superlattices, Physical Review Letters 61 (001) (1988) 24722475. doi:10.1103/PhysRevLett.61.2472.

[23] W. Z. G. Binasch, P. Griinberg, F. Saurenbach, Enhanced magnetoresistance in layered magnetic structures with antiferromagnetic interlayer exchange, Physical Review B 39 (7) (1989) 4828-4830.

[24] M. Julliere, Tunneling between ferromagnetic films, Physics Letters 54A (3) (1975) 225-226. doi:10.1016/0375-9601(75)90174-7. 
[25] T. Miyazaki, N. Tezuka, Giant Magnetic Tunneling Effect in $\mathrm{Fe} / \mathrm{Al} 2 \mathrm{O} 3 / \mathrm{Fe}$ Junction, Journal of Magnetism and Magnetic Materials 139 (1995) L231-L234.

[26] J. S. Moodera, L. R. Kinder, T. M. Wong, R. Meservey, Large magnetoresistance at room temperature in ferromagnetic thin film tunnel junctions, Physical Review Letters 74 (16) (1995) 3273-3276. doi:10.1103/PhysRevLett.74.3273.

[27] J. Mathon, A. Umerski, Theory of tunneling magnetoresistance of an epitaxial $\mathrm{Fe} / \mathrm{MgO} / \mathrm{Fe}(001)$ junction, Physical Review B Condensed Matter and Materials Physics 63 (22) (2001) 1-4. doi:10.1103/PhysRevB.63.220403.

[28] S. Yuasa, T. Nagahama, A. Fukushima, Y. Suzuki, K. Ando, Giant room-temperature magnetoresistance in single-crystal $\mathrm{Fe} / \mathrm{MgO} / \mathrm{Fe}$ magnetic tunnel junctions, Nature Materials 3 (12) (2004) 868-871. doi:10.1038/nmat1257.

[29] S. S. Parkin, C. Kaiser, A. Panchula, P. M. Rice, B. Hughes, M. Samant, S. H. Yang, Giant tunnelling magnetoresistance at room temperature with $\mathrm{MgO}$ (100) tunnel barriers, Nature Materials 3 (12) (2004) 862-867. doi:10.1038/nmat1256.

[30] S. Ikeda, J. Hayakawa, Y. Ashizawa, Y. M. Lee, K. Miura, H. Hasegawa, M. Tsunoda, F. Matsukura, H. Ohno, Tunnel magnetoresistance of $604 \%$ at $300 \mathrm{~K}$ by suppression of Ta diffusion in CoFeB$\mathrm{MgOCoFeB}$ pseudo-spin-valves annealed at high temperature, Applied Physics Letters 93 (8) (2008) 1-4. doi:10.1063/1.2976435.

[31] S. A. Wolf, D. D. Awschalom, R. A. Buhrman, J. M. Daughton, S. von Molnár, M. L. Roukes, A. Y. Chtchelkanova, D. M. Treger, Spintronics: A Spin-Based Electronics Vision for the Future, Science 294 (November) (2001) 1488.

[32] H. S. Wong, S. Salahuddin, Memory leads the way to better computing, Nature Nanotechnology 10 (3) (2015) 191-194. doi:10.1038/nnano.2015.29. 
[33] S. Tehrani, J. M. Slaughter, E. Chen, M. Durlam, J. Shi, M. DeHerrera, Progress and Outlook for MRAM Technology, IEEE Transactions on Magnetics 35 (5) (1999) 2814-2819.

[34] X. Dong, X. Wu, H. Li, Circuit and Microarchitecture Evaluation of 3D stacking magnetic RAM (MRAM) as a Universal Memory Replacement, in: Design Automation Conference, 2008, pp. 554-559.

[35] H. Zhao, B. Glass, P. K. Amiri, A. Lyle, Y. Zhang, Y. J. Chen, G. Rowlands, P. Upadhyaya, Z. Zeng, J. A. Katine, J. Langer, K. Galatsis, H. Jiang, K. L. Wang, I. N. Krivorotov, J. P. Wang, Sub-200ps spin transfer torque switching in in-plane magnetic tunnel junctions with interface perpendicular anisotropy, Journal of Physics D: Applied Physics 45 (2). doi:10.1088/0022-3727/45/2/025001.

[36] G. E. Rowlands, T. Rahman, J. A. Katine, J. Langer, A. Lyle, H. Zhao, J. G. Alzate, A. A. Kovalev, Y. Tserkovnyak, Z. M. Zeng, H. W. Jiang, K. Galatsis, Y. M. Huai, P. K. Amiri, K. L. Wang, I. N. Krivorotov, J. P. Wang, Deep subnanosecond spin torque switching in magnetic tunnel junctions with combined in-plane and perpendicular polarizers, Applied Physics Letters 98 (10). doi:10.1063/1.3565162.

[37] R. Patel, X. Guo, Q. Guo, E. Ipek, E. G. Friedman, Reducing Switching Latency and Energy in STT-MRAM Caches with Field-Assisted Writing, IEEE Transactions on Very Large Scale Integration (VLSI) Systems 24 (1) (2016) 129-138. doi:10.1109/TVLSI.2015.2401577.

[38] H. Liu, D. Bedau, D. Backes, J. A. Katine, J. Langer, A. D. Kent, Ultrafast switching in magnetic tunnel junction based orthogonal spin transfer devices, Applied Physics Letters 97 (24). doi:10.1063/1.3527962.

[39] Z. M. Zeng, P. Khalili Amiri, G. Rowlands, H. Zhao, I. N. Krivorotov, J. P. Wang, J. A. Katine, J. Langer, K. Galatsis, K. L. Wang, H. W. Jiang, Effect of resistance-area product on spin-transfer switching in MgO-based magnetic tunnel junction memory cells, Applied Physics Letters 98 (7). doi:10.1063/1.3556615.

[40] G. Jan, Y. J. Wang, T. Moriyama, Y. J. Lee, M. Lin, T. Zhong, R. Y. Tong, T. Torng, P. K. Wang, High spin torque efficiency of magnetic 
tunnel junctions with $\mathrm{MgO} / \mathrm{CoFeB} / \mathrm{MgO}$ free layer, Applied Physics Express 5 (9) (2012) 10-13. doi:10.1143/APEX.5.093008.

[41] E. Kitagawa, S. Fujita, K. Nomura, H. Noguchi, K. Abe, K. Ikegami, T. Daibou, Y. Kato, C. Kamata, S. Kashiwada, N. Shimomura, J. Ito, H. Yoda, Impact of ultra low power and fast write operation of advanced perpendicular MTJ on power reduction for high-performance mobile CPU, Technical Digest - International Electron Devices Meeting, IEDM (2012) 29.4.1-29.4.4doi:10.1109/IEDM.2012.6479129.

[42] K. Garello, C. O. Avci, I. M. Miron, M. Baumgartner, A. Ghosh, S. Auffret, O. Boulle, G. Gaudin, P. Gambardella, Ultrafast magnetization switching by spin-orbit torques, Applied Physics Letters 105 (21). doi:10.1063/1.4902443.

[43] M. Kazemi, G. E. Rowlands, S. Shi, R. A. Buhrman, E. G. Friedman, All-Spin-Orbit Switching of Perpendicular Magnetization, IEEE Transactions on Electron Devices 63 (11) (2016) 4499-4505. doi:10.1109/TED.2016.2604215.

[44] V. Sverdlov, A. Makarov, S. Selberherr, Reliable Sub-Nanosecond Switching of a Perpendicular SOT-MRAM Cell without External Magnetic Field, Proceedings of The 22nd World Multi-Conference on Systemics, Cybernetics and Informatics 16 (2) (2018) 55-59.

[45] J. G. Alzate, P. K. Amiri, P. Upadhyaya, S. S. Cherepov, J. Zhu, M. Lewis, R. Dorrance, J. A. Katine, J. Langer, K. Galatsis, D. Markovic, I. Krivorotov, K. L. Wang, Voltage-induced switching of nanoscale magnetic tunnel junctions, in: Technical Digest - International Electron Devices Meeting, IEDM, IEEE, 2012, pp. 681-684. doi:10.1109/IEDM.2012.6479130.

[46] P. K. Amiri, J. G. Alzate, X. Q. Cai, F. Ebrahimi, Q. Hu, K. Wong, C. Grèzes, H. Lee, G. Yu, X. Li, M. Akyol, Q. Shao, J. A. Katine, J. Langer, B. Ocker, K. L. Wang, Electric-Field-Controlled Magnetoelectric RAM: Progress, Challenges, and Scaling, IEEE Transactions on Magnetics 51 (11). doi:10.1109/TMAG.2015.2443124.

[47] C. Grezes, H. Lee, A. Lee, S. Wang, F. Ebrahimi, X. Li, K. Wong, J. A. Katine, B. Ocker, J. Langer, P. Gupta, P. Khalili Amiri, K. L. 
Wang, Write Error Rate and Read Disturbance in Electric-FieldControlled Magnetic Random-Access Memory, IEEE Magnetics Letters 8. doi:10.1109/LMAG.2016.2630667.

[48] A. Kirilyuk, A. V. Kimel, T. Rasing, Laser-induced magnetization dynamics and reversal in ferrimagnetic alloys, Reports on Progress in Physics 76 (2). doi:10.1088/0034-4885/76/2/026501.

[49] J. Walowski, M. Münzenberg, Perspective: Ultrafast magnetism and $\mathrm{THz}$ spintronics, Journal of Applied Physics 120 (14). arXiv:1606.08725, doi:10.1063/1.4958846.

[50] A. Stupakiewicz, K. Szerenos, D. Afanasiev, A. Kirilyuk, A. V. Kimel, Ultrafast nonthermal photo-magnetic recording in a transparent medium, Nature 542 (2017) 71-74. doi:10.1038/nature20807.

[51] A. Stupakiewicz, K. Szerenos, M. D. Davydova, K. A. Zvezdin, A. K. Zvezdin, A. Kirilyuk, A. V. Kimel, Selection rules for all-optical magnetic recording in iron garnet, Nature Communications 10 (612). doi:10.1038/s41467-019-08458-w.

URL http://dx.doi.org/10.1038/s41467-019-08458-w

[52] K. Szerenos, A. V. Kimel, A. Maziewski, A. Kirilyuk, A. Stupakiewicz, Fundamental Limits on the Repetition Rate of Photomagnetic Recording, Physical Review Applied 12 (044057). doi:10.1103/PhysRevApplied.12.044057.

URL https://doi.org/10.1103/PhysRevApplied.12.044057

[53] A. V. Kimel, All-optical switching: Three rules of design, Nature Materials 13 (3) (2014) 225-226. doi:10.1038/nmat3886.

[54] U. Atxitia, J. Barker, R. W. Chantrell, O. Chubykalo-Fesenko, Controlling the polarity of the transient ferromagneticlike state in ferrimagnets, Physical Review B 89 (22) (2014) 1-10. doi:10.1103/PhysRevB.89.224421.

[55] C. E. Graves, A. H. Reid, T. Wang, B. Wu, S. de Jong, K. Vahaplar, I. Radu, D. P. Bernstein, M. Messerschmidt, L. Müller, R. Coffee, M. Bionta, S. W. Epp, R. Hartmann, N. Kimmel, G. Hauser, A. Hartmann, P. Holl, H. Gorke, J. H. Mentink, A. Tsukamoto, A. Fognini, 
J. J. Turner, W. F. Schlotter, D. Rolles, H. Soltau, L. Strüder, Y. Acremann, A. V. Kimel, A. Kirilyuk, T. Rasing, J. Stöhr, A. O. Scherz, H. A. Dürr, Nanoscale spin reversal by non-local angular momentum transfer following ultrafast laser excitation in ferrimagnetic GdFeCo., Nature Materials 12 (4) (2013) 293-298. doi:10.1038/nmat3597.

[56] J. Y. Chen, L. He, J. P. Wang, M. Li, All-Optical Switching of Magnetic Tunnel Junctions with Single Subpicosecond Laser Pulses, Physical Review Applied 7 (2) (2017) 2-7. doi:10.1103/PhysRevApplied.7.021001.

[57] M. L. Lalieu, M. J. Peeters, S. R. Haenen, R. Lavrijsen, B. Koopmans, Deterministic all-optical switching of synthetic ferrimagnets using single femtosecond laser pulses, Physical Review B 96 (22) (2017) 1-5. doi:10.1103/PhysRevB.96.220411.

[58] J. H. Mentink, J. Hellsvik, D. V. Afanasiev, B. A. Ivanov, A. Kirilyuk, A. V. Kimel, O. Eriksson, M. I. Katsnelson, T. Rasing, Ultrafast spin dynamics in multisublattice magnets, Physical Review Letters 108 (5) (2012) 1-5. doi:10.1103/PhysRevLett.108.057202.

[59] D. Steil, S. Alebrand, A. Hassdenteufel, M. Cinchetti, M. Aeschlimann, All-optical magnetization recording by tailoring optical excitation parameters, Physical Review B 84 (22) (2011) 1-8. doi:10.1103/PhysRevB.84.224408.

[60] K. Vahaplar, A. M. Kalashnikova, A. V. Kimel, S. Gerlach, D. Hinzke, U. Nowak, R. Chantrell, A. Tsukamoto, A. Itoh, A. Kirilyuk, T. Rasing, All-optical magnetization reversal by circularly polarized laser pulses: Experiment and multiscale modeling, Physical Review B 85 (10) (2012) 1-17. doi:10.1103/PhysRevB.85.104402.

[61] G. Malinowski, F. Dalla Longa, J. H. Rietjens, P. V. Paluskar, R. Huijink, H. J. Swagten, B. Koopmans, Control of speed and efficiency of ultrafast demagnetization by direct transfer of spin angular momentum, Nature Physics 4 (11) (2008) 855-858. doi:10.1038/nphys1092.

[62] A. Melnikov, I. Razdolski, T. O. Wehling, E. T. Papaioannou, V. Roddatis, P. Fumagalli, O. Aktsipetrov, A. I. Lichtenstein, U. Bovensiepen, Ultrafast transport of laser-excited spin-polarized carriers 
in $\mathrm{Au} / \mathrm{Fe} / \mathrm{MgO}(001)$, Physical Review Letters 107 (7) (2011) 1-5. doi:10.1103/PhysRevLett.107.076601.

[63] A. Alekhin, I. Razdolski, N. Ilin, J. P. Meyburg, D. Diesing, V. Roddatis, I. Rungger, M. Stamenova, S. Sanvito, U. Bovensiepen, A. Melnikov, Femtosecond Spin Current Pulses Generated by the Nonthermal Spin-Dependent Seebeck Effect and Interacting with Ferromagnets in Spin Valves, Physical Review Letters 119 (1) (2017) 1-6. doi:10.1103/PhysRevLett.119.017202.

[64] N. Bergeard, M. Hehn, S. Mangin, G. Lengaigne, F. Montaigne, M. L. Lalieu, B. Koopmans, G. Malinowski, Hot-Electron-Induced Ultrafast Demagnetization in Co/Pt Multilayers, Physical Review Letters 117 (14) (2016) 1-5. doi:10.1103/PhysRevLett.117.147203.

[65] G. M. Choi, B. C. Min, Laser-driven spin generation in the conduction bands of ferrimagnetic metals, Physical Review B 97 (1) (2018) 8-12. doi:10.1103/PhysRevB.97.014410.

[66] M. Battiato, K. Carva, P. M. Oppeneer, Theory of laser-induced ultrafast superdiffusive spin transport in layered heterostructures, Physical Review B - Condensed Matter and Materials Physics 86 (2) (2012) 1-16. doi:10.1103/PhysRevB.86.024404.

[67] T. Roth, A. J. Schellekens, S. Alebrand, O. Schmitt, D. Steil, B. Koopmans, M. Cinchetti, M. Aeschlimann, Temperature dependence of laser-induced demagnetization in $\mathrm{Ni}$ : A key for identifying the underlying mechanism, Physical Review X 2 (2) (2012) 1-7. doi:10.1103/PhysRevX.2.021006.

[68] J. Kimling, J. Kimling, R. B. Wilson, B. Hebler, M. Albrecht, D. G. Cahill, Ultrafast demagnetization of $\mathrm{FePt}: \mathrm{Cu}$ thin films and the role of magnetic heat capacity, Physical Review B Condensed Matter and Materials Physics 90 (22) (2014) 1-9. doi:10.1103/PhysRevB.90.224408.

[69] M. Battiato, K. Carva, P. M. Oppeneer, Superdiffusive spin transport as a mechanism of ultrafast demagnetization, Physical Review Letters 105 (2) (2010) 1-4. doi:10.1103/PhysRevLett.105.027203. 
[70] G. Malinowski, N. Bergeard, M. Hehn, S. Mangin, Hot-electron transport and ultrafast magnetization dynamics in magnetic multilayers and nanostructures following femtosecond laser pulse excitation, European Physical Journal B 91: 98. doi:10.1140/epjb/e2018-80555-5.

[71] A. J. Schellekens, K. C. Kuiper, R. R. J. C. De Wit, B. Koopmans, Ultrafast spin-transfer torque driven by femtosecond pulsed-laser excitation, Nature Communications 5 (2014) 1-7. doi:10.1038/ncomms5333.

[72] G. M. Choi, B. C. Min, K. J. Lee, D. G. Cahill, Spin current generated by thermally driven ultrafast demagnetization, Nature Communications 5 (2014) 1-8. doi:10.1038/ncomms5334.

[73] J. Kimling, D. G. Cahill, Spin diffusion induced by pulsed-laser heating and the role of spin heat accumulation, Physical Review B 95 (1) (2017) 014402. doi:10.1103/PhysRevB.95.014402.

[74] E. Iacocca, T. M. Liu, A. H. Reid, Z. Fu, S. Ruta, P. W. Granitzka, E. Jal, S. Bonetti, A. X. Gray, C. E. Graves, R. Kukreja, Z. Chen, D. J. Higley, T. Chase, L. Le Guyader, K. Hirsch, H. Ohldag, W. F. Schlotter, G. L. Dakovski, G. Coslovich, M. C. Hoffmann, S. Carron, A. Tsukamoto, A. Kirilyuk, A. V. Kimel, T. Rasing, J. Stöhr, R. F. Evans, T. Ostler, R. W. Chantrell, M. A. Hoefer, T. J. Silva, H. A. Dürr, Spin-current-mediated rapid magnon localisation and coalescence after ultrafast optical pumping of ferrimagnetic alloys, Nature Communications 10 (1). doi:10.1038/s41467-019-09577-0.

[75] W. Wang, D. G. Cahill, Limits to thermal transport in nanoscale metal bilayers due to weak electron-phonon coupling in $\mathrm{Au}$ and $\mathrm{Cu}$, Physical Review Letters 109 (17) (2012) 1-5. doi:10.1103/PhysRevLett.109.175503.

[76] S. Bonetti, M. C. Hoffmann, M. J. Sher, Z. Chen, S. H. Yang, M. G. Samant, S. S. Parkin, H. A. Dürr, THz-Driven Ultrafast Spin-Lattice Scattering in Amorphous Metallic Ferromagnets, Physical Review Letters 117 (8) (2016) 1-5. doi:10.1103/PhysRevLett.117.087205.

[77] R. Wilson, S. Coh, How long, and why, do Photoexcited Electrons in a Metal Remain Hot?, ArXiv e-printsarXiv:1906.11397. 
[78] W. S. Fann, R. Storz, H. W. Tom, J. Bokor, Electron thermalization in gold, Physical Review B 46 (20) (1992) 13592-13595. doi:10.1103/PhysRevB.46.13592.

[79] W. S. Fann, R. Storz, H. W. Tom, J. Bokor, Direct measurement of nonequilibrium electron-energy distributions in sub-picosecond laserheated gold films, Physical Review Letters 68 (18) (1992) 2834-2837. doi:10.1016/0039-6028(93)90985-S.

[80] K. Mistry, C. Allen, C. Auth, B. Beattie, D. Bergstrom, M. Bost, M. Brazier, M. Buehler, A. Cappellani, R. Chau, C. Choi, G. Ding, K. Fischer, T. Ghani, R. Grover, W. Han, D. Hanken, M. Hattendorf, J. He, J. Hicks, R. Huessner, D. Ingerly, P. Jain, R. James, L. Jong, S. Joshi, C. Kenyon, K. Kuhn, K. Lee, H. Liu, J. Maiz, B. Mcintyre, P. Moon, J. Neirynck, S. Pae, C. Parker, D. Parsons, C. Prasad, L. Pipes, M. Prince, P. Ranade, T. Reynolds, J. Sandford, L. Shifren, J. Sebastian, J. Seiple, D. Simon, S. Sivakumar, P. Smith, C. Thomas, T. Troeger, P. Vandervoorn, S. Williams, K. Zawadzki, A 45nm Logic Tech with HKMG Transistors, Strained Si, $9 \mathrm{Cu}$ Interconnect Layers, 193 nm Dry Patterning, and 100\% Pb-free Packaging, in: IEEE International Electron Devices Meeting, 2007, pp. 247-250.

[81] J. Gorchon, C. H. Lambert, Y. Yang, A. Pattabi, R. B. Wilson, S. Salahuddin, J. Bokor, Single shot ultrafast all optical magnetization switching of ferromagnetic Co/Pt multilayers, Applied Physics Letters 111 (4) (2017) 042401. doi:10.1109/E3S.2017.8246170.

[82] P. J. Metaxas, J. P. Jamet, J. Ferré, B. Rodmacq, B. Dieny, R. L. Stamps, Magnetic domain wall creep in the presence of an effective interlayer coupling field, Journal of Magnetism and Magnetic Materials 320 (21) (2008) 2571-2575. doi:10.1016/j.jmmm.2008.03.041.

[83] Z. Y. Liu, F. Zhang, N. Li, B. Xu, J. L. He, D. L. Yu, Y. J. Tian, Oscillatory antiferromagnetic interlayer coupling in Co $(4 \AA)$ $\mathrm{Pt}(\mathrm{tPt} \AA)[\mathrm{Co}(4 \AA) \mathrm{Pt}(6 \AA)$ Co $(4 \AA)] \mathrm{NiO}(20 \AA)$ multilayers with perpendicular anisotropy, Physical Review B 77 (1) (2008) 1-4. doi:10.1103/PhysRevB.77.012409.

[84] X. X. Li, J. Bao, L. Y. Lu, X. G. Xu, Y. Jiang, Oscillatory antiferromagnetic interlayer coupling in $\mathrm{Co} / \mathrm{Pt}$ multilayer with perpendicu- 
lar anisotropy, Solid State Communications 148 (5-6) (2008) 209-212. doi:10.1016/j.ssc.2008.08.017.

[85] M. Bersweiler, D. Lacour, K. Dumesnil, F. Montaigne, M. Hehn, Phase diagram in exchange-coupled $\mathrm{CoTb} /[\mathrm{Co} / \mathrm{Pt}]$ multilayer-based magnetic tunnel junctions, Physical Review B 92 (22) (2015) 1-11. doi:10.1103/PhysRevB.92.224431.

[86] R. Schafer, Magneto-optical domain studies in coupled magnetic multilayers, Journal of Magnetism and Magnetic Materials 148 (1995) 226231.

[87] M. Finazzi, M. Savoini, A. R. Khorsand, A. Tsukamoto, A. Itoh, L. Duò, A. Kirilyuk, T. Rasing, M. Ezawa, Laser-induced magnetic nanostructures with tunable topological properties, Physical Review Letters 110 (17) (2013) 1-5. doi:10.1103/PhysRevLett.110.177205.

[88] T. M. Liu, T. Wang, A. H. Reid, M. Savoini, X. Wu, B. Koene, P. Granitzka, C. E. Graves, D. J. Higley, Z. Chen, G. Razinskas, M. Hantschmann, A. Scherz, J. Stöhr, A. Tsukamoto, B. Hecht, A. V. Kimel, A. Kirilyuk, T. Rasing, H. A. Dürr, Nanoscale Confinement of All-Optical Magnetic Switching in TbFeCo - Competition with Nanoscale Heterogeneity, Nano Letters 15 (10) (2015) 6862-6868. doi:10.1021/acs.nanolett.5b02743.

[89] L. L. Guyader, S. E. Moussaoui, E. Mengotti, L. J. Heyderman, F. Nolting, A. Tsukamoto, A. Itoh, A. Kirilyuk, T. Rasing, A. V. Kimel, Nanostructuring of GdFeCo Thin Films for Laser Induced Magnetization Switching, Journal of the Magnetics Society of Japan 36 (1_2) (2011) 21-23. doi:10.3379/msjmag.1108m005.

[90] L. Le Guyader, S. El Moussaoui, M. Buzzi, R. V. Chopdekar, L. J. Heyderman, A. Tsukamoto, A. Itoh, A. Kirilyuk, T. Rasing, A. V. Kimel, F. Nolting, Demonstration of laser induced magnetization reversal in GdFeCo nanostructures, Applied Physics Letters 101 (2). doi:10.1063/1.4733965.

[91] A. El-Ghazaly, B. Tran, A. Ceballos, C.-H. Lambert, A. Pattabi, S. Salahuddin, F. Hellman, J. Bokor, Ultrafast magnetization switching 
in nanoscale magnetic dots, Applied Physics Letters 114 (23) (2019) 232407. doi:10.1063/1.5098453.

[92] A. Mekonnen, A. R. Khorsand, M. Cormier, A. V. Kimel, A. Kirilyuk, A. Hrabec, L. Ranno, A. Tsukamoto, A. Itoh, T. Rasing, Role of the inter-sublattice exchange coupling in short-laser-pulse-induced demagnetization dynamics of GdCo and GdCoFe alloys, Physical Review B 87 (18) (2013) 2-6. doi:10.1103/PhysRevB.87.180406.

[93] L. Le Guyader, M. Savoini, S. El Moussaoui, M. Buzzi, A. Tsukamoto, A. Itoh, A. Kirilyuk, T. Rasing, a. V. Kimel, F. Nolting, Nanoscale sub-100 picosecond all-optical magnetization switching in GdFeCo microstructures, Nature Communications 6 (5839). arXiv:1407.4010, doi:10.1038/ncomms6839.

[94] M. Wietstruk, A. Melnikov, C. Stamm, T. Kachel, N. Pontius, M. Sultan, C. Gahl, M. Weinelt, H. A. Dürr, U. Bovensiepen, Hot-electrondriven enhancement of spin-lattice coupling in $\mathrm{Gd}$ and $\mathrm{Tb} 4 \mathrm{f}$ ferromagnets observed by femtosecond x-ray magnetic circular dichroism, Physical Review Letters 106 (12) (2011) 3-6. arXiv:1010.1374, doi:10.1103/PhysRevLett.106.127401.

[95] T. Q. Qiu, C. L. Tien, Size Effects on Nonequilibrium Laser Heating of Metal Films, Journal of Heat Transfer 115 (4) (1993) 842-847. doi:10.1115/1.2911378.

[96] J. L. Hostetler, A. N. Smith, D. M. Czajkowsky, P. M. Norris, Measurement of the electron-phonon coupling factor dependence on film thickness and grain size in $\mathrm{Au}, \mathrm{Cr}$, and $\mathrm{Al}$, Applied Optics 38 (16) (1999) 3614. doi:10.1364/AO.38.003614.

[97] P. Corkum, F. Brunel, N. Sherman, T. Srinivasan-Rao, Thermal response of metals to ultrashort-pulse laser excitation., Physical review letters 61 (25) (1988) 2886-2889. doi:10.1103/PhysRevLett.61.2886.

[98] D. C. Ralph, M. D. Stiles, Spin transfer torques, Journal of Magnetism and Magnetic Materials 320 (7) (2008) 1190-1216. arXiv:0711.4608, doi:10.1016/j.jmmm.2007.12.019. 
[99] G. Th., V. d. B. H.A.M., H. J., B. L., R. Th., Ultrafast precessional magnetization reversal by picosecond magnetic field pulse shaping, Nature 418 (6897) (2002) 509-512.

[100] I. M. Miron, G. Gaudin, S. Auffret, B. Rodmacq, A. Schuhl, S. Pizzini, J. Vogel, P. Gambardella, Current-driven spin torque induced by the Rashba effect in a ferromagnetic metal layer, Nature Materials 9 (3) (2010) 230-234. doi:10.1038/nmat2613.

[101] K. Jhuria, J. Hohlfeld, A. Pattabi, E. Martin, A. Y. A. Córdova, X. Shi, R. Lo Conte, S. Petit-Watelot, J. C. Rojas-Sanchez, G. Malinowski, S. Mangin, A. Lemaître, M. Hehn, J. Bokor, R. B. Wilson, J. Gorchon, Picosecond Spin Orbit Torque Switching, ArXiv eprintsarXiv:1912.01377.

[102] M. Bibes, A. Barthélémy, Towards a magnetoelectric memory, Nature Materials 7 (6) (2008) 425-426. doi:10.1038/nmat2189.

[103] P. B. Meisenheimer, S. Novakov, N. M. Vu, J. T. Heron, Perspective: Magnetoelectric switching in thin film multiferroic heterostructures, Journal of Applied Physics 123 (24). doi:10.1063/1.5031446.

[104] J. Ko, J. Hong, Voltage-Assisted Magnetic Switching in MgO/CoFeBBased Magnetic Tunnel Junctions by Way of Interface Reconstruction, ACS Applied Materials and Interfaces 9 (48) (2017) 42296-42301. doi:10.1021/acsami.7b11293.

[105] S. H. C. Baek, K. W. Park, D. S. Kil, Y. Jang, J. Park, K. J. Lee, B. G. Park, Complementary logic operation based on electric-field controlled spin-orbit torques, Nature Electronics 1 (7) (2018) 398-403. doi:10.1038/s41928-018-0099-8.

[106] G. Zhang, W. Hübner, E. Beaurepaire, J.-Y. Bigot, Laser-induced ultrafast demagnetization: Femtomagnetism, a new frontier?, in: B. Hillebrands, K. Ounadjela (Eds.), Spin Dynamics in Confined Magnetic Structures I, Springer Berlin Heidelberg, Berlin, Heidelberg, 2002, pp. 245-289.

[107] J.-Y. Bigot, M. Vomir, Ultrafast magnetization dynamics of nanostructures, Annalen der Physik 525 (1-2) (2013) 2-30. doi:10.1002/andp.201200199. 